HETEROCYCLES, Vol. , No. , , pp. -. (C) The Japan Institute of Heterocyclic Chemistry Received, , Accepted, , Published online,
DOI: 10.3987/COM- (Please do not delete.)

\title{
RAPID STEREOSELECTIVE SYNTHESES OF HETEROARENE-FUSED AZACYCLES VIA DIASTEREOSELECTIVE CONJUGATE ADDITION OF HETEROARYL SUBSTITUTED LITHIUM AMIDES $\dagger$
}

\section{Stephen G. Davies,* Katherine E. Holder, Ai M. Fletcher, Paul M. Roberts, James E. Thomson and David Zimmer}

Department of Chemistry, Chemistry Research Laboratory, University of Oxford, Mansfield Road, Oxford, OX1 3TA, U.K.

\begin{abstract}
Conjugate addition of heteroaryl substituted lithium amides to a range of $\alpha, \beta$-unsaturated esters followed by in situ enolate oxidation with (-)-(camphorsulfonyl)oxaziridine gave the corresponding $\alpha$-hydroxy- $\beta$-amino esters. Subsequent Friedel-Crafts type cyclisation of these $\alpha$-hydroxy- $\beta$-amino esters gave a range of heteroarene-fused azacycles in good yields and high diastereoselectivities.
\end{abstract}

\section{INTRODUCTION}

Arene- and heteroarene-fused azacyclic compounds have been shown to display potent biological activities. For example, (S)-salsolinol 1 is a clinical treatment for cancer, ${ }^{1}$ and A-86929 2 and ABT-431 3 have been used for treatment of Parkinson's disease. ${ }^{2}$ Very recently, ORC-13661 4 was reported as a promising drug candidate for the prevention of aminoglycoside induced hearing loss (Figure 1). ${ }^{3}$ Thus, the efficient synthesis and biological evaluation of various analogues of these classes of molecules would benefit drug discovery chemistry significantly.
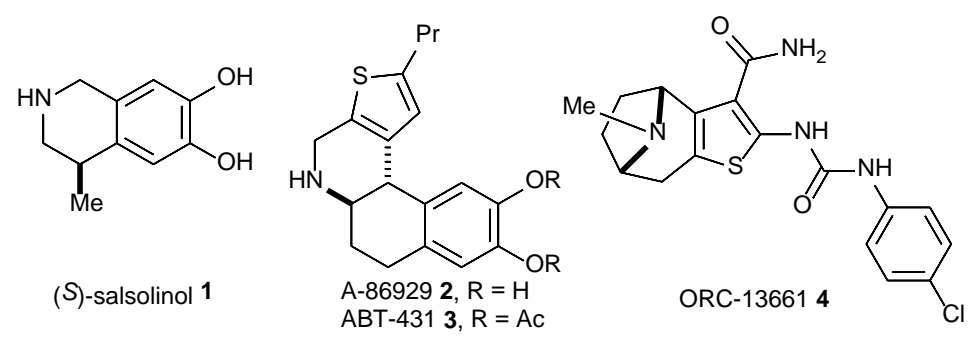

Figure 1 The structures of biologically active arene and heteroarene-fused cyclic compounds $\mathbf{1}-\mathbf{4}$.

\footnotetext{
* Corresponding Author. E-mail: steve.davies@chem.ox.ac.uk

† Dedicated to Professor Tohru Fukuyama on the occasion of his 70th birthday.
} 
We have recently reported the efficient stereoselective syntheses of 1,2,3,4-tetrahydroisoquinolines (i.e., arene-fused azacyclic compounds) via the intramolecular Friedel-Crafts type alkylation of the benzylic carbenium ion intermediates derived from anti- $\alpha$-hydroxy- $\beta$-amino esters. ${ }^{4}$ For example, treatment of $\alpha$-hydroxy- $\beta$-amino ester 5 with $\mathrm{Tf}_{2} \mathrm{O}$ and 2,6-di-tert-butyl-4-methylpyridine gave 1,2,3,4-tetrahydroisoquinoline $\mathbf{9}$ in 72\% yield as a single diastereoisomer. This outcome is consistent with a proposed mechanism involving activation of the hydroxyl group within $\mathbf{5}$ as the corresponding triflate $\mathbf{6}$, displacement by the adjacent amino group to give aizirdinium intermediate 7 [with inversion of configuration at $\mathrm{C}(2)$ ], and subsequent rupture of the $\mathrm{C}(3)-\mathrm{N}$ bond forming the corresponding benzylic carbenium ion 8, which undergoes rapid Friedel-Crafts type cyclisation to give 1,2,3,4-tetrahydroisoquinoline $\mathbf{9}$ (Scheme 1).

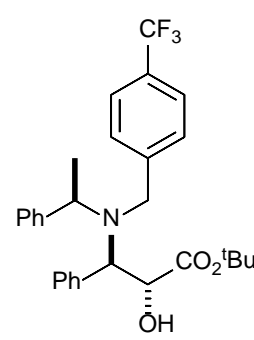

5, >99:1 dr

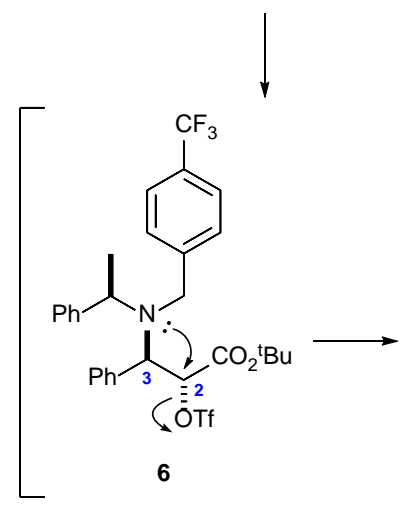

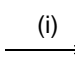

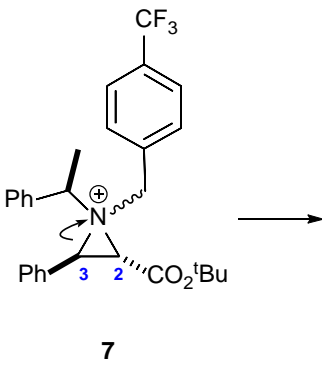

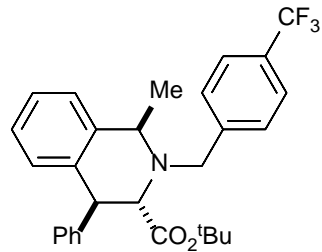

9, $72 \%,>99: 1 \mathrm{dr}$

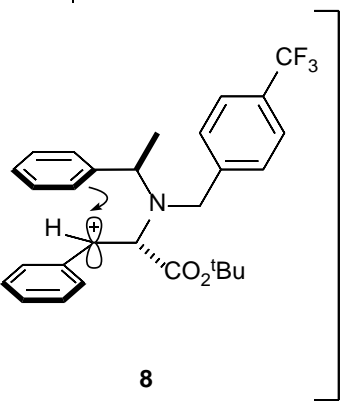

Scheme 1 Reagents and conditions: (i) $\mathrm{Tf}_{2} \mathrm{O}, 2$,6-di-tert-butyl-4-methylpyridine, $\mathrm{CH}_{2} \mathrm{Cl}_{2}, 0{ }^{\circ} \mathrm{C}$ to rt, $6 \mathrm{~h}$.

In order to develop further this methodology and to expand the structural diversity of the resultant arene-fused azabicyclic products accessible, $\alpha$-hydroxy- $\beta$-amino esters 12 , bearing a variety of heteroarylmethyl $N$-substituents were recognised as cyclisation precursors. It was envisaged that $\alpha$-hydroxy- $\beta$-amino esters 12 could be synthesised via the conjugate addition of the corresponding heteroaryl substituted lithium amides $\mathbf{1 1}$ to $\alpha, \beta$-unsaturated esters $\mathbf{1 0}$ followed by in situ enolate oxidation with (camphorsulfonyl)oxaziridine (CSO) $14 .^{5}$ The resultant $\alpha$-hydroxy- $\beta$-amino esters 12, incorporating the corresponding heteroaryl motif, would then be exposed to Friedel-Crafts alkylation type conditions to 
allow access to novel bicyclic molecular architectures 13 (Figure 2). Herein, we report our investigations in this area.

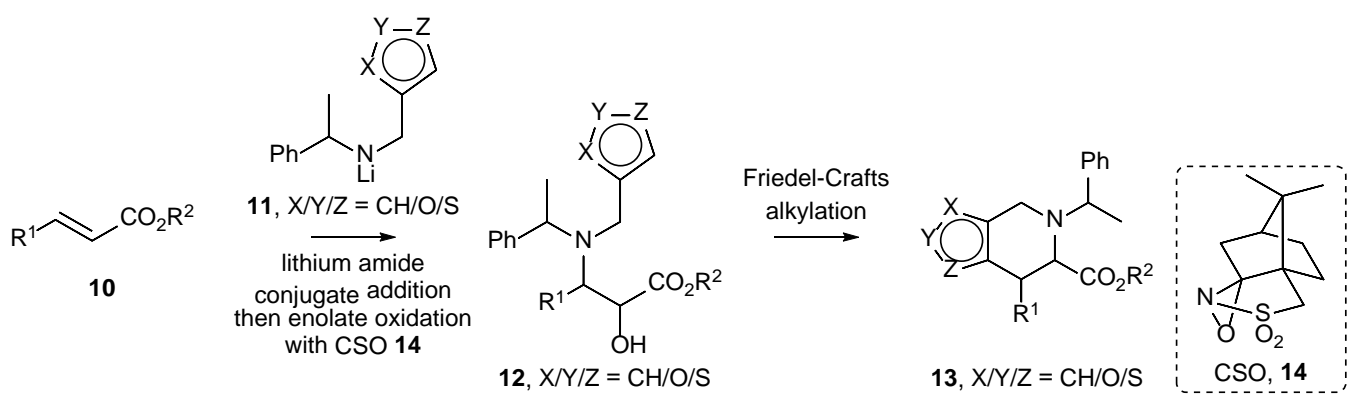

Figure 2 Proposed synthetic route to heteroarene-fused azabicycles.

\section{RESULTS AND DISCUSSION}

A range of heteroaryl substituted amines [derived from $(R S)$ - $\alpha$-methylbenzylamine] were prepared via a standard reductive alkylation procedure upon reaction with the requisite aldehydes. ${ }^{6}$ Commercially available aldehydes 15-18 were treated with racemic $\alpha$-methylbenzylamine $(R S)-\mathbf{1 9}$ and subsequent reduction with $\mathrm{NaBH}_{4}$ gave secondary amines (RS)-20-23 in 77-85\% yield (Scheme 2).

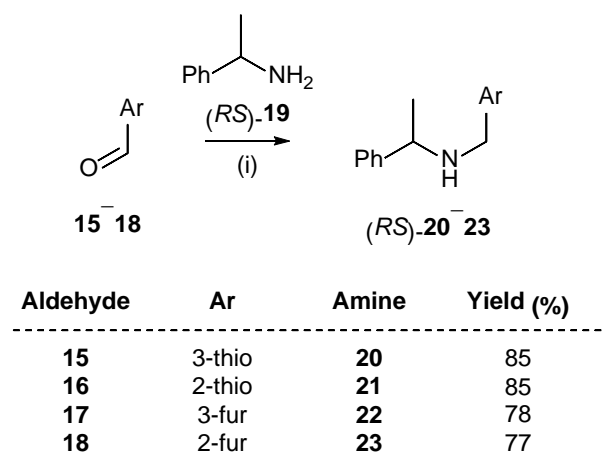

Scheme 2 Reagents and conditions: (i) (RS)-19, EtOH, rt, 24 h then $\mathrm{NaBH}_{4}, 0{ }^{\circ} \mathrm{C}$ to rt, $48 \mathrm{~h}$. [thio $=$ thiophenyl; fur $=$ furyl $]$.

Using an one-pot procedure, addition of $s$-BuLi to a mixture of amines (RS)-20-23 and $\alpha, \beta$-unsaturated ester 24 gave the corresponding lithium amides $(R S)$-25-28 in situ, which underwent conjugate addition to $\alpha, \beta$-unsaturated ester 24: conjugate addition of 3-thiophenyl, 2-thiophenyl and 3-furyl substituted lithium amides (RS)-25-27 to $\alpha, \beta$-unsaturated ester $\mathbf{2 4}$ gave the corresponding $\beta$-amino esters $\mathbf{2 9 - 3 1}$ in $80 \%, 69 \%$ and $89 \%$ yield, respectively, as single diasteroisomers ( $>95: 5 \mathrm{dr}$ ) in each case. However, conjugate addition of 2-furyl substituted lithium amide (RS)-28 to $\alpha, \beta$-unsaturated ester $\mathbf{2 4}$ gave the corresponding $\beta$-amino ester 32 in 77\% yield and 62:38 dr (Scheme 3). The low diastereoselectivity observed upon conjugate addition of the 2-furyl substituted lithium amide reagent $(R S)-\mathbf{2 8}$ to $\mathbf{2 4}$ is presumably due to disruption of the normal mode of chelation in the transition state ${ }^{7}$ by the proximal oxygen atom of the 2-furyl group, 
resulting in a non-selective pathway for conjugate addition. Similar phenomena have been observed previously when heteroatoms (such as nitrogen and oxygen atoms) were present in either the lithium amide reagent or the $\alpha, \beta$-unsaturated ester, giving rise to a reduction in diastereoselectivity. ${ }^{8,9,10}$ The corresponding reaction with 2-thiophenyl substituted lithium amide (RS)-26 gave high diastereoselectivity ( $>95: 5 \mathrm{dr}$ ), presumably due to the lower lithium chelating ability of the sulfur atom.

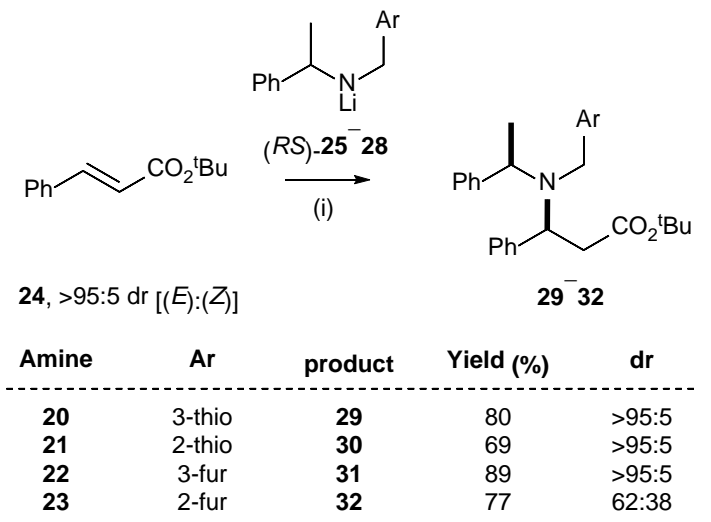

Scheme 3 Reagents and conditions: (i) (RS)-20-23, s-BuLi, THF, $-78{ }^{\circ} \mathrm{C}, 2 \mathrm{~h}$.

$$
\text { [thio }=\text { thiophenyl; fur }=\text { furyl }] \text {. }
$$

The relative configuration within the major diastereoisomeric $\beta$-amino ester product $\mathbf{3 1}$ was assigned by chemical correlation to the known $\beta$-amino ester $(3 S, \alpha R)-34,{ }^{11}$ which was obtained upon conjugate addition of lithium (R)- $N$-benzyl- $N$-( $\alpha$-methylbenzyl)amide $(R)$-33 to $\alpha, \beta$-unsaturated ester $\mathbf{2 4}$ in $98 \%$ yield as a single diastereoisomer. The $(3 S, \alpha R)$-configuration within $\mathbf{3 4}$ has previously been established via single crystal X-ray diffraction analysis. ${ }^{12}$ Treatment of $(3 S, \alpha R)$-34 with cerium ammonium nitrate (CAN) promoted chemoselective mono- $N$-debenzylation ${ }^{13}$ to give secondary $\beta$-amino ester (3S, $\left.\alpha R\right)$-35 in $87 \%$ yield. An authentic sample of 3-furyl substituted $\beta$-amino ester (3S, $\alpha R)$-31 was obtained upon $N$-alkylation of $(3 S, \alpha R)$-35 with the requisite bromide 36, which was prepared from the corresponding alcohol under Appel conditions (Scheme 4). The spectroscopic data for the enantiopure sample of (3S, $\alpha R)$-31 were identical to those for the sample of (3RS, $\alpha S R)$-31 derived from conjugate addition of 3-furyl substituted lithium amide $(R S)$-27 to $\alpha, \beta$-unsaturated ester 24. Thus, this unambiguously established the relative configuration within 31. The relative configurations within the major diastereoisomeric $\beta$-amino ester products 29, 30 and 32 were assigned by analogy to that of $\mathbf{3 1}$. 


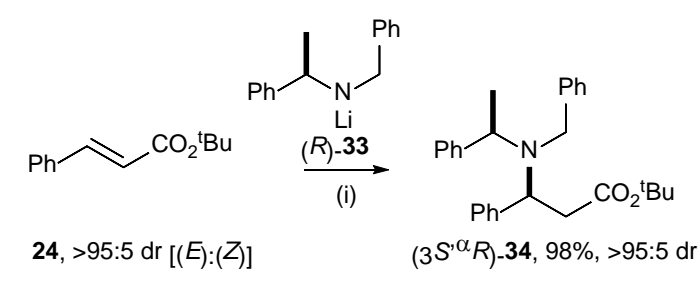

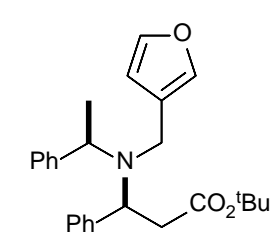

$\left(3 S^{\prime \alpha}\right)-31,28 \%,>95: 5 d^{a}$

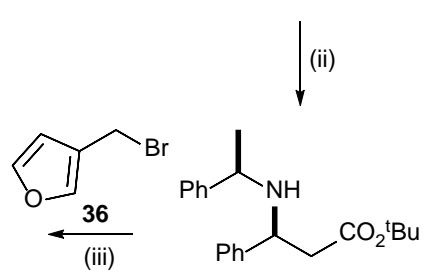

$\left(3^{,}{ }^{, \alpha} R\right)-35,87 \%,>95: 5 d r$

Scheme 4 Reagents and conditions: (i) (R)-33, THF, $-78^{\circ} \mathrm{C}, 2 \mathrm{~h}$; (ii) CAN, $\mathrm{MeCN} / \mathrm{H}_{2} \mathrm{O}$ (5:1), rt, 2 h; (iii) 36, rt, 16 h. ${ }^{\text {a }}$ Isolated in $>80 \%$ purity.

The conjugate addition of 3-furyl substituted lithium amide (RS)-27, as a representative heteroaryl substituted lithium amide reagent, to a range of $C(3)$-aryl substituted $\alpha, \beta$-unsaturated esters 37-40 was next investigated. In all cases, the corresponding $\beta$-amino esters $\mathbf{4 1 - 4 4}$ were obtained as single diastereoisomers (>95:5 dr) in 49-85\% yield (Scheme 5). The relative configurations within $\mathbf{4 1 - 4 4}$ were assigned by analogy to $\mathrm{C}(3)$-phenyl substituted $\beta$-amino ester 31.

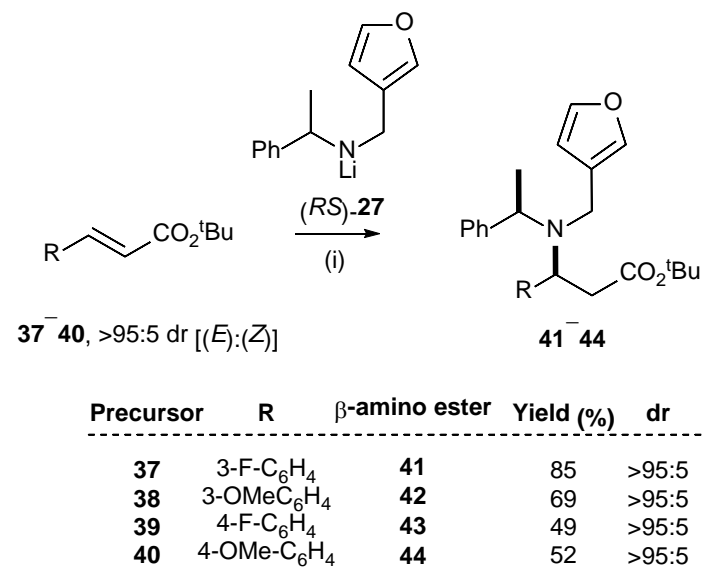

Scheme 5 Reagents and conditions: (i) (RS)-22, s-BuLi, THF, $-78{ }^{\circ} \mathrm{C}, 2 \mathrm{~h}$.

$\alpha, \beta$-Unsaturated ester 24 was next treated with $N$-heteroaryl substituted lithium amides (RS)-25-27 followed by subsequent addition of (-)-CSO 14, from which the corresponding anti- $\alpha$-hydroxy- $\beta$-amino esters 45-47 were obtained in 48-58\% yield and $\geq 94: 6 \mathrm{dr}$ (Scheme 6). ${ }^{9}$ The relative 2,3-anti-configurations within $\alpha$-hydroxy- $\beta$-amino esters 45-47 were assigned by analogy to other aminohydroxylation reactions using lithium $N$-benzyl- $N$-( $\alpha$-methylbenzyl)amide 33 and CSO $14^{9}$ and were supported by the diagnostic values of the ${ }^{1} \mathrm{H}$ NMR ${ }^{3} J$ coupling constants $\left({ }^{3} J_{2,3}=2.9-3.5 \mathrm{~Hz}\right)$ between the $\mathrm{C}(2) H$ and $\mathrm{C}(3) H$ protons. ${ }^{14,15}$ 


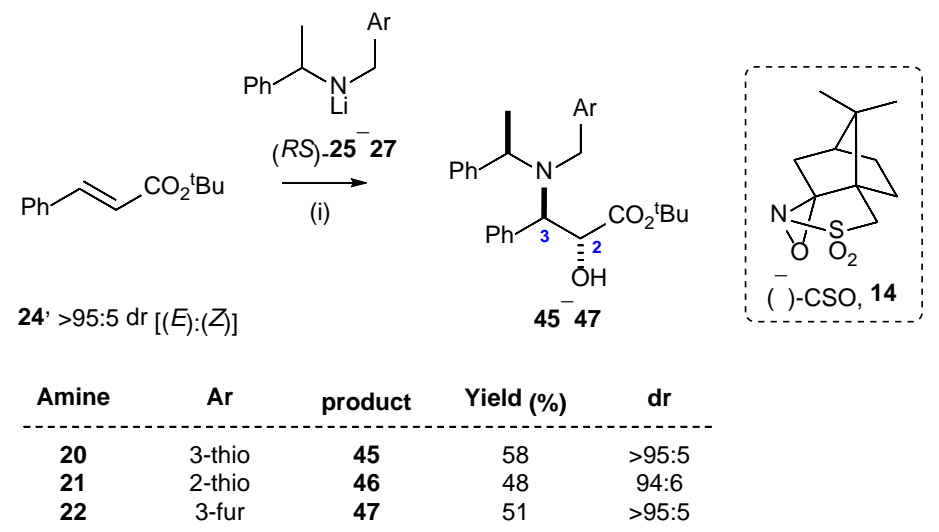

Scheme 6 Reagents and conditions: (i) (RS)-20-22, s-BuLi, THF, $-78{ }^{\circ} \mathrm{C}, 2 \mathrm{~h}$ then (-)-CSO 14, $-78{ }^{\circ} \mathrm{C}$ to rt, $16 \mathrm{~h}$. [thio = thiophenyl; fur $=$ furyl $]$.

The aminohydroxylation of a range of $\alpha, \beta$-unsaturated esters 37-40 upon reaction with 3-furyl substituted lithium amide (RS)-27 and (-)-CSO 14 was also examined: conjugate addition of lithium amide (RS)-27 to $\alpha, \beta$-unsaturated esters 37-40 followed by in situ enolate oxidation with (-)-CSO 14 gave the corresponding anti- $\alpha$-hydroxy- $\beta$-amino esters $\mathbf{4 8 - 5 1}$ in 38-46\% yield (Scheme 7). The relative configurations within 4851 were again assigned by analogy to other aminohydroxylation reactions using lithium $N$-benzyl- $N$-( $\alpha$-methylbenzyl)amide 33 and CSO $14^{9}$ and were supported by the diagnostic values of the ${ }^{1} \mathrm{H}$ NMR ${ }^{3} J_{2,3}$ coupling constants $\left({ }^{3} J_{2,3}=3.0-3.2 \mathrm{~Hz}\right)$.

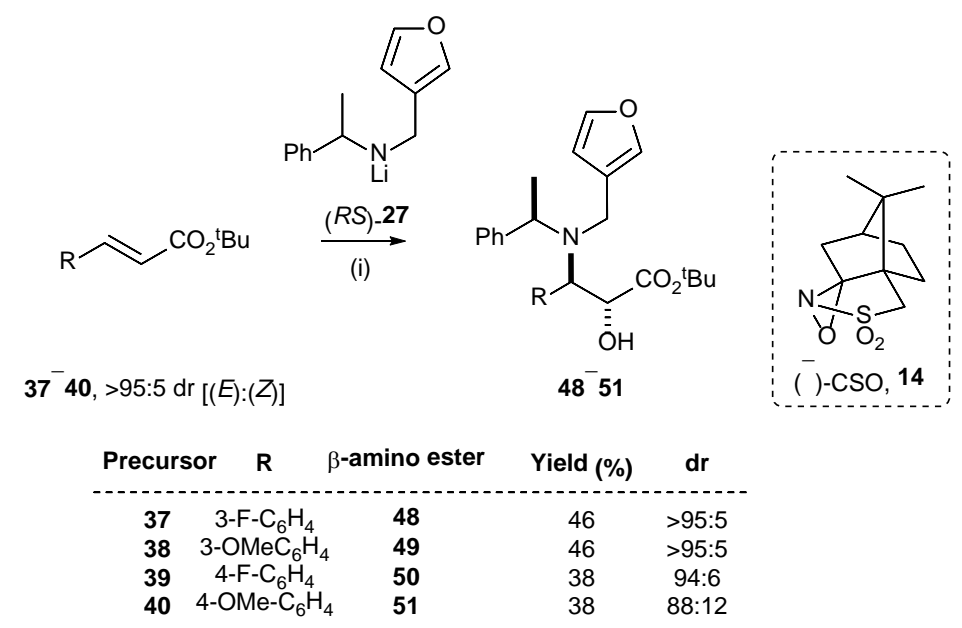

Scheme 7 Reagents and conditions: (i) 22, s-BuLi, THF, $-78{ }^{\circ} \mathrm{C}, 2 \mathrm{~h}$ then (-)-CSO 14, $-78{ }^{\circ} \mathrm{C}$ to rt, $16 \mathrm{~h}$.

The Friedel-Crafts alkylation type cyclisation protocol employed in our previous syntheses of 1,2,3,4-tetrahydroisoquinolines ${ }^{15}$ was applied to $N$-heteroaryl substituted $\alpha$-hydroxy- $\beta$-amino esters $\mathbf{4 5}$ and 47-51: substrates 45 and 47-51 were treated with $\mathrm{Tf}_{2} \mathrm{O}$ and 2,6-di-tert-butyl-4-methylpyiridine in $\mathrm{CH}_{2} \mathrm{Cl}_{2}$ to give 4,5,6,7-tetrahydrothieno[3,2-c]pyridine 55 and 4,5,6,7-tetrahydrofura[3,2-c]pyridines 56-60 in 42$81 \%$ yield and $\geq 88: 12 \mathrm{dr}$ in each case. The relative configurations within 55-60 were tentatively assigned 
from the ${ }^{1} \mathrm{H}$ NMR ${ }^{3} J$ coupling constants between the stereogenic C(6)H and C(7)H protons of the heteroaryl fused tetrahydropyridines 55-60 $\left({ }^{3} J_{6,7}=1.9-2.5 \mathrm{~Hz}\right)$ by analogy to those shown to be diagnostic for the corresponding 6,7-anti-disubstituted 1,2,3,4-tetrahydroisoquinolines. ${ }^{16}$ The formation of 4,5,6,7-tetrahedrothieno[3,2-c]pyridine $\mathbf{5 5}$ and 4,5,6,7-tetrahydrofura[3,2-c]pyridines $\mathbf{5 6 - 6 0}$ and the stereochemical outcomes of these reactions is consistent with the proposed mechanism for 1,2,3,4-tetrahydroisoquinoline formation: treatment of $\mathbf{4 5}$ and $\mathbf{4 7 - 5 1}$ with $\mathrm{Tf}_{2} \mathrm{O}$ activates the hydroxyl group as the corresponding triflate 52 and intramolecular displacement of the resultant triflate by the amino group [with inversion of configuration of $\mathrm{C}(2)$ ] forms the corresponding aziridinium intermediate 53. Regioselective ring-opening of 53 gave carbenium ion 54, which was rapidly trapped by the most nucleophilic $\mathrm{C}(2)$ position of the $N$-hereoarene ring ${ }^{17}$ [with retention of configuration at $\mathrm{C}(3)$ ] to give the bicyclic compounds 55-60 (Scheme 8).

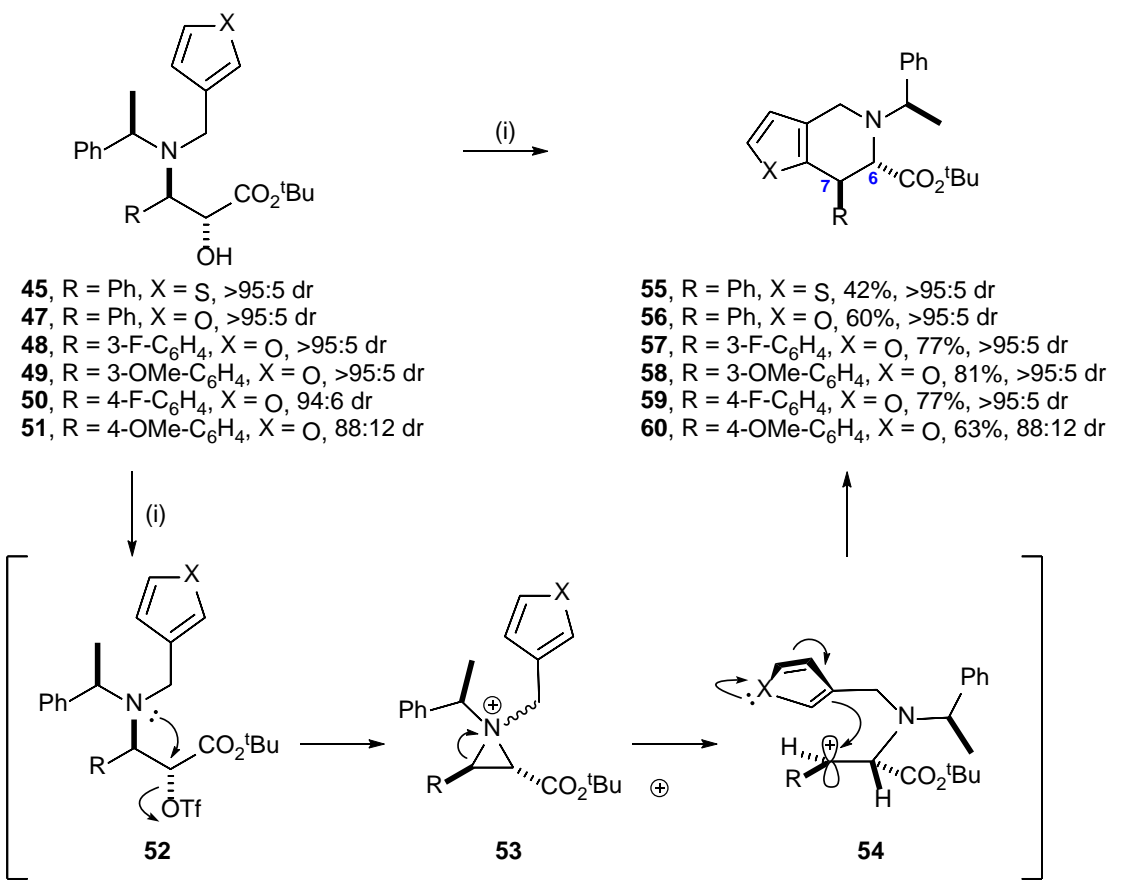

Scheme 8 Reagents and conditions: (i) $\mathrm{Tf}_{2} \mathrm{O}$, 2,6-di-tert-butyl-4-methylpyridine, $\mathrm{CH}_{2} \mathrm{Cl}_{2}, 0{ }^{\circ} \mathrm{C}$ to rt, $6 \mathrm{~h}$.

The preparation of these heteroarene-fused azacycles in enantiopure form could be readily achieved by employing the analogous synthetic route, however using enantiopure heteroaryl substituted lithium amide reagent in the conjugate addition step. As a representative example, enantiopure amine $(R)$-22 [prepared in 82\% yield from $(R)$ - $\alpha$-methylbenzyl amine $(R)$-19] was reacted with $\alpha, \beta$-unsaturated ester 24 followed by $\mathrm{NH}_{4} \mathrm{Cl}$, which gave the corresponding $\beta$-amino ester $(3 S, \alpha R)-31$ in $62 \%$ and $>95: 5 \mathrm{dr}$. The corresponding aminohydroxylation of 24 with (R)-22 and (-)-CSO 14 gave $\alpha$-hydroxy- $\beta$-amino ester $(R, R, R)-47$ in $50 \%$ yield and >95:5 dr. The Friedel-Crafts type cyclisation protocol applied to $(R, R, R)-\mathbf{4 7}$ gave enantiopure heteroarene-fused azacycle $(6 S, 7 R, \alpha R)-56$ in 64\% and >95:5 dr (Scheme 9). 


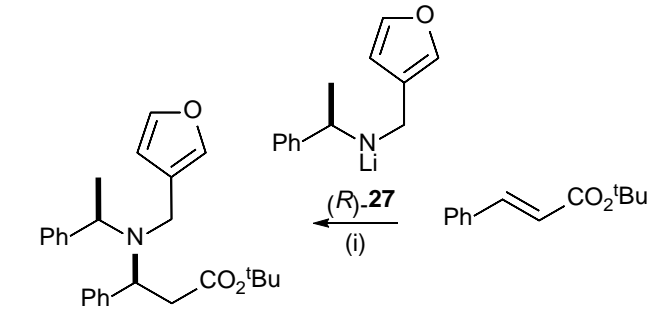

$\left({ }^{\prime} S^{\prime \alpha} R\right)-31,62 \%,>95: 5 d r$

25, >95:5 dr $[(E):(Z)]$

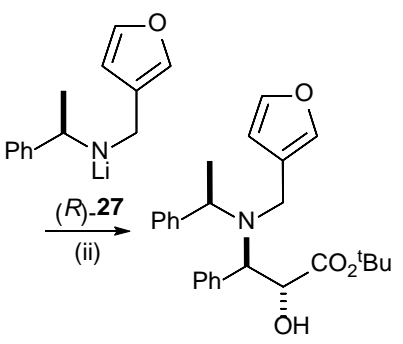

$\left(R^{\prime} R^{\prime} R\right)-47,50 \%,>95: 5 d r$

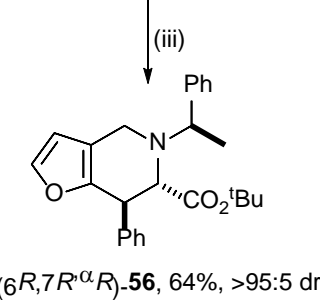

Scheme 9 Reagents and conditions: (i) (R)-22, s-BuLi, THF, $-78{ }^{\circ} \mathrm{C}, 2 \mathrm{~h}$; (ii) (R)-22, s-BuLi, THF, $-78{ }^{\circ} \mathrm{C}$, $2 \mathrm{~h}$ then (-)-CSO $14,-78{ }^{\circ} \mathrm{C}$ to rt, $16 \mathrm{~h}$; (iii) $\mathrm{Tf}_{2} \mathrm{O}$, 2,6-di-tert-butyl-4-methylpyridine, $\mathrm{CH}_{2} \mathrm{Cl}_{2}, 0^{\circ} \mathrm{C}$ to rt, $6 \mathrm{~h}$.

In conclusion, the stereoselective syntheses of several heteroaryl fused azacyclic compounds were demonstrated. Conjugate addition of thiophenyl and furyl substituted lithium amides to a range of $\alpha, \beta$-unsaturated esters gave the corresponding $\beta$-amino esters and the configuration of newly formed stereogenic centre within the $\beta$-amino esters were established via chemical correlation. Conjugate addition of heteroaryl substituted lithium amides to $\alpha, \beta$-unsaturated esters and subsequent in situ enolate oxidation with (-)-(camphorsulfonyl)oxaziridine gave the corresponding anti- $\alpha$-hydroxy- $\beta$-amino esters with high diastereoselectivity. Cyclisation of the resultant anti- $\alpha$-hydroxy- $\beta$-amino esters via Friedel-Crafts type cyclisation protocol gave a range of heteroaryl fused azacyclic compounds in good yield and high diastereoselectivity. The application of this strategy for the preparation of enantiopure heteroarene-fused azacycles was also demonstrated in one representative case.

\section{EXPERIMENTAL}

Reactions involving organometallic or other moisture-sensitive reagents were carried out under a nitrogen or argon atmosphere using standard vacuum line techniques and glassware that was flame dried and cooled under nitrogen before use. Solution of $n$-BuLi in hexanes and $s$-BuLi in cyclohexane were purchased and titrated against diphenylacetic acid before use. Solvents were dried according to the procedure outlined by Grubbs and co-workers. ${ }^{18}$ Water was purified by an Elix ${ }^{\circledR}$ UV-10 system. All other reagents were used as supplied (analytical or HPLC grade) without prior purification. Organic layers were dried over $\mathrm{MgSO}_{4}$ or $\mathrm{NaSO}_{4}$. Thin layer chromatography was performed on aluminium plates coated with $60 \mathrm{~F}_{254}$ silica. Plates were visualised using UV light (254 nm), iodine, 1\% aq $\mathrm{KMnO}_{4}$, or 10\% ethanolic phosphomolybdic acid. Flash column chromatography was performed on Kieselgel 60 silica. 
Melting points were recorded on a Gallenkamp Hot Stage apparatus. Optical rotations were recorded on a Perkin-Elmer 241 polarimeter with a water-jacketed $10 \mathrm{~cm}$ cell. Specific rotations are reported in $10^{-1}$ deg $\mathrm{cm}^{2} \mathrm{~g}^{-1}$ and concentrations in $\mathrm{g} / 100 \mathrm{~mL}$. IR spectra were recorded on a Bruker Tensor 27 FT-IR spectrometer using an ATR module. Selected characteristic peaks are reported in $\mathrm{cm}^{-1}$. NMR spectra were recorded on Bruker Avance spectrometers in the deuterated solvent stated. Spectra were recorded at rt. The field was locked by external referencing to the relevant deuteron resonance. ${ }^{1} \mathrm{H}-{ }^{1} \mathrm{H}$ COSY,${ }^{1} \mathrm{H}-{ }^{13} \mathrm{C}$ HSQC, and ${ }^{1} \mathrm{H}-{ }^{13} \mathrm{C}$ HMBC analyses were used to establish atom connectivity. Low-resolution mass spectra were recorded on either a VG MassLab 20-250 or a Micromass Platform 1 spectrometer. Accurate mass measurements were run on either a Bruker MicroTOF internally calibrated with polyalanine, or a Micromass GCT instrument fitted with a Scientific Glass Instruments BPX5 column (15 $\mathrm{m} \times 0.25 \mathrm{~mm}$ ) using amyl acetate as a lock mass .

General procedure 1 - Preparation of secondary amines: The requisite aryl carboxaldehyde (1.05 equiv) was added to a stirred solution of $(R S)$ - $\alpha$-methylbenzylamine (1.00 equiv) in EtOH (1.77 M with respect to amine). The resultant mixture was stirred at rt for $24 \mathrm{~h}$ before being cooled to $0{ }^{\circ} \mathrm{C}$. $\mathrm{NaBH}_{4}$ equiv) was then added and the resultant suspension was stirred at rt for $48 \mathrm{~h}$. The resultant suspension concentrated in vacuo and the residue was partitioned between $10 \%$ aq citric acid solution and $\mathrm{CH}_{2} \mathrm{Cl}_{2}$. aqueous layer was extracted with $\mathrm{CH}_{2} \mathrm{Cl}_{2}$, and the combined aqueous extracts were neutralised with $2.0 \mathrm{M}$ aq $\mathrm{NaOH}$, extracted with $\mathrm{CH}_{2} \mathrm{Cl}_{2}$, and washed with brine, then dried and concentrated in vacuo.

General procedure 2 - Lithium amide conjugate addition: s-BuLi (1.4 M in cyclohexane, 1.55 equiv) was added dropwise via syringe to a stirred solution of the requisite secondary amine (1.60 equiv) and the requisite $\alpha, \beta$-unsaturated ester (1.00 equiv) in THF $\left(0.4 \mathrm{M}\right.$ with respect to amine) at $-78{ }^{\circ} \mathrm{C}$. The reaction mixture was stirred at $-78{ }^{\circ} \mathrm{C}$ for $2 \mathrm{~h}$ before the addition of satd aq $\mathrm{NH}_{4} \mathrm{Cl}$. The resultant mixture was allowed to warm to rt over 15 min then concentrated in vacuo. The residue was then partitioned between $\mathrm{CH}_{2} \mathrm{Cl}_{2}$ and $10 \%$ aq citric acid solution. The aqueous layer was extracted with $\mathrm{CH}_{2} \mathrm{Cl}_{2}$ and the combined organic extracts were washed sequentially with satd aq $\mathrm{NaHCO}_{3}, \mathrm{H}_{2} \mathrm{O}$ and brine, then dried and concentrated in vacuo.

General procedure 3 - Lithium amide conjugate addition with $\boldsymbol{\alpha}$-hydroxylation: $s$-BuLi (1.4 M in cyclohexane, 1.55 equiv) was added dropwise via syringe to a stirred solution of the requisite secondary amine (1.60 equiv) and the requisite $\alpha, \beta$-unsaturated ester (1.00 equiv) in THF ( $0.4 \mathrm{M}$ with respect to amine) at $-78{ }^{\circ} \mathrm{C}$. The resultant solution was stirred at $-78{ }^{\circ} \mathrm{C}$ for $2 \mathrm{~h}$. (-)-CSO 14 (1.6 equiv) was then added and the reaction mixture was allowed to warm to rt, then stirred at rt for $18 \mathrm{~h}$. Satd aq $\mathrm{NH}_{4} \mathrm{Cl}$ was added and the reaction mixture was stirred at rt for $5 \mathrm{~min}$, then concentrated in vacuo. The residue was then partitioned between $\mathrm{CH}_{2} \mathrm{Cl}_{2}$ and $10 \%$ aq citric acid solution. The aqueous layer was extracted with 
$\mathrm{CH}_{2} \mathrm{Cl}_{2}$ and the combined organic extracts were washed sequentially with satd aq $\mathrm{NaHCO}_{3}, \mathrm{H}_{2} \mathrm{O}$ and brine, then dried and concentrated in vacuo.

General procedure 4 - Rearrangement/Friedel-Crafts alkylation: $\mathrm{Tf}_{2} \mathrm{O}$ (1.5 equiv) was added to a stirred solution of the requisite $\alpha$-hydroxy- $\beta$-amino ester $(1.0$ equiv) and 2,6-di-tert-butyl-4-methylpyridine (3.0 equiv) in $\mathrm{CH}_{2} \mathrm{Cl}_{2}(0.08 \mathrm{M}$ with respect to $\alpha$-hydroxy- $\beta$-amino ester) at $0{ }^{\circ} \mathrm{C}$, and the resultant mixture was stirred at rt for $6 \mathrm{~h} . \mathrm{H}_{2} \mathrm{O}$ was then added and the aqueous layer was extracted with $\mathrm{CH}_{2} \mathrm{Cl}_{2}$. The combined organic extracts were then dried and concentrated in vacuo. The residue was dissolved in $\mathrm{Et}_{2} \mathrm{O}$ then the resultant solution was filtered and concentrated in vacuo.

(RS)- $N$-(Thiophen-3-ylmethyl)- $N$-( $\alpha$-methylbenzyl)amine 20: Following General procedure 1, 15 (7.81 $\mathrm{mL}, 89.3 \mathrm{mmol})$ was reacted with $(R S)-19(10.9 \mathrm{~mL}, 85.0 \mathrm{mmol})$ in $\mathrm{EtOH}(48.0 \mathrm{~mL})$ then $\mathrm{NaBH}_{4}(3.23 \mathrm{~g}$, $85.0 \mathrm{mmol})$ to give 20 as a pale yellow oil (15.8 g, 85\%); $v_{\max }(\mathrm{ATR}) 3330(\mathrm{~N}-\mathrm{H}) ; \delta_{\mathrm{H}}\left(400 \mathrm{MHz}, \mathrm{CDCl}_{3}\right)$ 1.38 (3H, d, $J$ 6.6, C $(\alpha) M e), 3.66$ (2H, app s, NCH $\mathrm{Ar}), 3.82(1 \mathrm{H}, \mathrm{q}, J$ 6.6, C $(\alpha) H), 7.03(1 \mathrm{H}, \mathrm{dd}, J$ 4.9, 0.9, C(5)H), 7.11 (1H, dd, J 1.8, 0.9, C(2)H), 7.24-7.38 (6H, m, Ph, C(4)H); $\delta_{\mathrm{C}}\left(100 \mathrm{MHz}, \mathrm{CDCl}_{3}\right) 24.4$ (C( $\alpha) M e), 46.6\left(\mathrm{NCH}_{2} \mathrm{Ar}\right), 57.5(C(\alpha)), 121.4(C(2))$, 125.6, 126.7, 126.9, 128.5 (C(4), o,m,p-Ph), 127.6 (C(5)), $141.6(C(3)), 145.4(\mathrm{i}-\mathrm{Ph}) ; \mathrm{m} / \mathrm{z}\left(\mathrm{ESI}^{+}\right) 218\left([\mathrm{M}+\mathrm{H}]^{+}, 100 \%\right) ; \mathrm{HRMS}\left(\mathrm{ESI}^{+}\right) \mathrm{C}_{13} \mathrm{H}_{16} \mathrm{NS}^{+}\left([\mathrm{M}+\mathrm{H}]^{+}\right)$ requires 218.0998; found 218.0999.

(RS)-N-(Thiophen-2-ylmethyl)- $N$-( $\alpha$-methylbenzyl)amine $21:^{19}$ Following General procedure 1,16 $(12.0 \mathrm{~mL}, 129 \mathrm{mmol})$ was reacted with $(R S)-19(15.7 \mathrm{~mL}, 122 \mathrm{mmol})$ in EtOH $(69.1 \mathrm{~mL})$ then $\mathrm{NaBH}_{4}$ (4.65 g, $122 \mathrm{mmol}$ ) to give 21 as a pale yellow oil (23.6 g, 85\%); $\delta_{\mathrm{H}}\left(400 \mathrm{MHz}, \mathrm{CDCl}_{3}\right) 1.39$ (3H, d, $J$ 6.7, $\mathrm{C}(\alpha) M e), 3.81\left(1 \mathrm{H}, \mathrm{d}, J\right.$ 14.3, $\left.\mathrm{NCH}_{\mathrm{A}} \mathrm{H}_{\mathrm{B}} \mathrm{Ar}\right), 3.86\left(1 \mathrm{H}, \mathrm{d}, J\right.$ 14.3, $\left.\mathrm{NCH}_{\mathrm{A}} H_{\mathrm{B}} \mathrm{Ar}\right), 3.87(1 \mathrm{H}, \mathrm{q}, J$ 6.7, $\mathrm{C}(\alpha) H)$, 6.87 (1H, dd, $J$ 3.4, 1.0, C(3)H), 6.95 (1H, dd, $J$ 5.1, 3.4, C(4)H), 7.21 (1H, dd, $J$ 5.1, 1.0, C(5)H) 7.25$7.38(5 \mathrm{H}, \mathrm{m}, \mathrm{Ph})$.

(RS)-N-(Furan-3-ylmethyl)- $N$-( $\alpha$-methylbenzyl)amine 22: ${ }^{20}$ Following General procedure 1, 17 (18.0 mL, $208 \mathrm{mmol})$ was reacted with $(R S)-19(25.2 \mathrm{~mL}, 198 \mathrm{mmol})$ in EtOH $(112 \mathrm{~mL})$ then $\mathrm{NaBH}_{4}(7.53 \mathrm{~g}$, $198 \mathrm{mmol}$ ) to give 22 as a pale yellow oil (31.1 g, 78\%); $\delta_{\mathrm{H}}\left(400 \mathrm{MHz}, \mathrm{CDCl}_{3}\right) 1.39$ (3H, d, J 6.6, $\mathrm{C}(\alpha) M e), 3.83\left(2 \mathrm{H}\right.$, app s, NCH$\left.{ }_{2} \mathrm{Ar}\right), 3.87(1 \mathrm{H}, \mathrm{q}, J$ 6.6, C $(\alpha) H), 6.38(1 \mathrm{H}, \mathrm{d}, J 0.9, \mathrm{C}(4) H), 7.25-7.39$ (7H, m, C(2)H, C(5)H, Ph).

(R)- $N$-(Furan-3-ylmethyl)- $N$-( $\boldsymbol{\alpha}$-methylbenzyl)amine 22: Following General procedure 1, 17 (1.80 mL, $20.8 \mathrm{mmol})$ was reacted with $(R)-\mathbf{1 9}(2.52 \mathrm{~mL}, 19.8 \mathrm{mmol})$ in EtOH $(11.7 \mathrm{~mL})$ then $\mathrm{NaBH}_{4}(754 \mathrm{mg}$, $19.8 \mathrm{mmol}$ ) to give $(R)-22$ as a pale yellow oil (3.28 g, 82\%); $[\alpha]_{\mathrm{D}}^{22}+45.9$ (c 1.0 in $\left.\mathrm{CHCl}_{3}\right)$.

(RS)-N-(Furan-2-ylmethyl)- $N$-( $\boldsymbol{\alpha}$-methylbenzyl)amine 23: ${ }^{20}$ Following General procedure 1, 18 (17.3 mL, $208 \mathrm{mmol})$ was reacted with $(R S)-19(25.2 \mathrm{~mL}, 198 \mathrm{mmol})$ in EtOH $(112 \mathrm{~mL})$ then $\mathrm{NaBH}_{4}(7.53 \mathrm{~g}$, 
$198 \mathrm{mmol}$ ) to give 23 as a pale yellow oil (30.6 g, 77\%); $\delta_{\mathrm{H}}\left(400 \mathrm{MHz}, \mathrm{CDCl}_{3}\right) 1.37$ (3H, d, $J$ 6.6, $\mathrm{C}(\alpha) M e), 3.59\left(1 \mathrm{H}, \mathrm{d}, J\right.$ 14.3, $\left.\mathrm{NCH}_{\mathrm{A}} \mathrm{H}_{\mathrm{B}} \mathrm{Ar}\right), 3.67$ (1H, d, $J$ 14.3, $\left.\mathrm{NCH}_{\mathrm{A}} H_{\mathrm{B}} \mathrm{Ar}\right), 3.79(1 \mathrm{H}, \mathrm{q}, J$ 6.6, C $(\alpha) H)$, 6.11 (1H, dd, J 3.1, 0.6, C(3)H), 6.31 (1H, J 3.1, 1.8, C(4)H), 7.24-7.38 (6H, m, C(5)H, Ph).

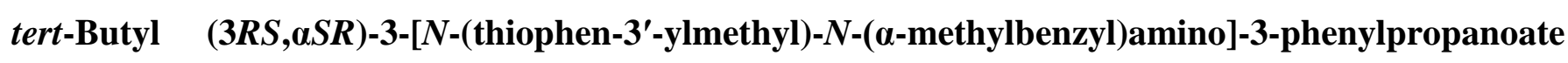
29: Following General procedure 2, s-BuLi (1.4 M in cyclohexane, $1.72 \mathrm{~mL}, 2.23 \mathrm{mmol})$ was reacted with 20 (500 mg, $2.30 \mathrm{mmol}$ ) and 24 (294 mg, $1.44 \mathrm{mmol}$, >95:5 dr [(E):(Z)]) in THF (5.76 mL). Purification via flash column chromatography (eluent $30-40{ }^{\circ} \mathrm{C}$ petrol/ $\mathrm{Et}_{2} \mathrm{O} / 35 \%$ aqNH${ }_{4} \mathrm{OH}, 90: 9: 1$ ) gave 29 as a pale yellow oil $(488 \mathrm{mg}, 80 \%,>95: 5 \mathrm{dr}) ; v_{\max }(\mathrm{ATR}) 1725(\mathrm{C}=\mathrm{O}) ; \delta_{\mathrm{H}}\left(400 \mathrm{MHz}, \mathrm{CDCl}_{3}\right) 1.24$ (9H, s, CMe3), 1.26 (3H, d, J 6.8, C( $\alpha) M e), 2.51$ (1H, dd, $J$ 14.7, 9.8, C(2)HA ), 2.58 (1H, dd, J 14.7, 5.2,

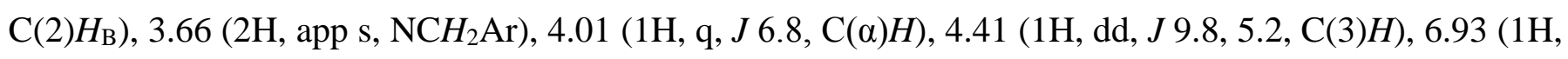
dd, $J$ 4.9, 1.2, C(4')H), 7.05 (1H, dd, $\left.J 2.9,1.2, \mathrm{C}\left(2^{\prime}\right) H\right), 7.19$ (1H, dd, $\left.J 4.9,2.9, \mathrm{C}\left(5^{\prime}\right) H\right), 7.21-7.43(10 \mathrm{H}$, m, Ph); $\delta_{\mathrm{C}}\left(100 \mathrm{MHz}, \mathrm{CDCl}_{3}\right) 16.2(\mathrm{C}(\alpha) M e), 27.8\left(\mathrm{CMe}_{3}\right), 38.7(C(2)), 46.2\left(\mathrm{NCH}_{2} \mathrm{Ar}\right), 57.0(C(\alpha)), 59.6$ (C(3)), 80.2 (CMe $), 121.2\left(C\left(2^{\prime}\right)\right), 125.1\left(C\left(5^{\prime}\right)\right), 127.1\left(C\left(4^{\prime}\right)\right), 126.8,127.7,127.9,128.1,128.2$ (o,m,p-Ph), 141.8 (i-Ph), $143.0\left(C\left(3^{\prime}\right)\right), 144.3$ (i-Ph), $171.1(C(1)) ; \mathrm{m} / \mathrm{z}\left(\mathrm{ESI}^{+}\right) 422\left([\mathrm{M}+\mathrm{H}]^{+}, 100 \%\right)$; HRMS $\left(\mathrm{ESI}^{+}\right) \mathrm{C}_{26} \mathrm{H}_{32} \mathrm{NO}_{2} \mathrm{~S}^{+}\left([\mathrm{M}+\mathrm{H}]^{+}\right)$requires 422.2148; found 422.2144.

tert-Butyl (3RS,aSR)-3-[N-(thiophen-2'-ylmethyl)- $N$-( $\alpha$-methylbenzyl)amino]-3-phenylpropanoate

30: Following General procedure 2, s-BuLi (1.4 M in cyclohexane, $1.51 \mathrm{~mL}, 196 \mathrm{mmol})$ was reacted with 21 (440 mg, $2.03 \mathrm{mmol}$ ) and 24 (295 mg, $1.27 \mathrm{mmol},>95: 5 \mathrm{dr}$ [(E):(Z)]) in THF (5.07 mL). Purification via flash column chromatography (eluent $30-40{ }^{\circ} \mathrm{C}$ petrol/Et $\mathrm{E}_{2} \mathrm{O} / 35 \%$ aqNH${ }_{4} \mathrm{OH}, 90: 9: 1$ ) gave 30 as a pale yellow oil (367 mg, 69\%, >95:5 dr); $v_{\max }(\mathrm{ATR}) 1726(\mathrm{C}=\mathrm{O}) ; \delta_{\mathrm{H}}\left(400 \mathrm{MHz}, \mathrm{CDCl}_{3}\right) 1.23\left(9 \mathrm{H}, \mathrm{s}, \mathrm{CMe}_{3}\right)$, 1.29 (3H, d, $J$ 6.9, C( $\alpha) M e$ ), 2.55 (1H, dd, $J$ 14.5, 9.8, C(2)H $\left.H_{\mathrm{A}}\right), 2.59$ (1H, dd, $J$ 14.5, 5.4, C(2)HB), 3.86 (2H, app s, $\left.\mathrm{NCH}_{2} \mathrm{Ar}\right), 4.06(1 \mathrm{H}, \mathrm{q}, J$ 6.9, $\mathrm{C}(\alpha) H), 4.45(1 \mathrm{H}, \mathrm{dd}, J$ 9.8, 5.4, C(3)H), 6.84-6.87 (1H, m, $\left.\mathrm{C}\left(3^{\prime}\right) H\right), 6.88\left(1 \mathrm{H}\right.$, dd, $J$ 5.0, 3.5, C(4')H), $7.16\left(1 \mathrm{H}, \mathrm{dd}, J\right.$ 5.0, 1.3, C(5')H), 7.22-7.47 (10H, m, Ph); $\delta_{\mathrm{C}}$ (100 MHz, $\left.\mathrm{CDCl}_{3}\right) 15.9(\mathrm{C}(\alpha) M e), 27.8\left(\mathrm{CMe}_{3}\right), 38.7(C(2)), 46.1\left(\mathrm{NCH}_{2} \mathrm{Ar}\right), 56.8(C(\alpha)), 59.5(C(3))$, $80.2\left(C \mathrm{Me}_{3}\right), 123.9\left(C\left(3^{\prime}\right)\right), 124.2\left(C\left(5^{\prime}\right)\right), 126.3\left(C\left(4^{\prime}\right)\right), 126.9,127.2,127.8,128.1,128.2(o, m, p-P h)$, 141.7 (i-Ph), $143.9(\mathrm{i}-\mathrm{Ph}), 147.0\left(C\left(2^{\prime}\right)\right), 171.1(C(1)) ; \mathrm{m} / \mathrm{z}\left(\mathrm{ESI}^{+}\right) 422\left([\mathrm{M}+\mathrm{H}]^{+}, 100 \%\right) ; \mathrm{HRMS}\left(\mathrm{ESI}^{+}\right)$ $\mathrm{C}_{26} \mathrm{H}_{32} \mathrm{NO}_{2} \mathrm{~S}^{+}\left([\mathrm{M}+\mathrm{H}]^{+}\right)$requires 422.2148; found 422.2145 .

tert-Butyl (3RS, $\alpha S R)$-3-[N-(furan-3'-ylmethyl)- $N$-( $\alpha$-methylbenzyl)amino]-3-phenylpropanoate 31: Following General procedure 2, s-BuLi (1.4 M in cyclohexane, $5.43 \mathrm{~mL} 7.60 \mathrm{mmol}$ ) was reacted with 22 (1.58 g, $7.84 \mathrm{mmol})$ and 24 (1.00 g, $4.90 \mathrm{mmol}$, >95:5 dr [(E):(Z)]) in THF (19.6 mL). Purification via flash column chromatography (eluent $30-40{ }^{\circ} \mathrm{C}$ petrol/Et ${ }_{2} \mathrm{O} / 35 \%$ aqNH$H_{4} \mathrm{OH}, 90: 9: 1$ ) gave 31 as a yellow oil (1.76 g, 89\%, >95:5 dr); v $v_{\max }(\mathrm{ATR}) 1725$ (C=O); $\delta_{\mathrm{H}}\left(400 \mathrm{MHz}, \mathrm{CDCl}_{3}\right) 1.25$ (9H, s, CMe $), 1.25$ (3H, d, $J$ 6.8, C $(\alpha) M e), 2.55\left(1 \mathrm{H}, \mathrm{dd}, J 14.6,9.8, \mathrm{C}(2) H_{\mathrm{A}}\right), 2.62\left(1 \mathrm{H}, \mathrm{dd}, J 14.6,5.2, \mathrm{C}(2) H_{\mathrm{B}}\right), 3.51(2 \mathrm{H}$, app s, $\left.\mathrm{NCH}_{2} \mathrm{Ar}\right), 4.03(1 \mathrm{H}, \mathrm{q}, J$ 6.8, C $(\alpha) H), 4.43\left(1 \mathrm{H}, \mathrm{dd}, J\right.$ 9.8, 5.2, C(3)H), $6.24\left(1 \mathrm{H}, \mathrm{dd}, J 1.7,0.8, \mathrm{C}\left(4^{\prime}\right) H\right)$, 
7.22-7.42 (12H, m, C(2')H, C(5')H,Ph); $\delta_{\mathrm{C}}\left(100 \mathrm{MHz}, \mathrm{CDCl}_{3}\right) 16.2(\mathrm{C}(\alpha) M e), 27.8\left(\mathrm{CMe}_{3}\right), 38.8(C(2))$, $41.5\left(\mathrm{NCH}_{2} \mathrm{Ar}\right), 56.6(C(\alpha)), 59.3(C(3)), 80.2\left(C \mathrm{Me}_{3}\right), 110.8\left(C\left(4^{\prime}\right)\right), 125.4\left(C\left(3^{\prime}\right)\right), 126.7,127.1,127.7$, 128.1, 128.2 (o,m,p-Ph), 139.9 (C(2')), 141.8 (i-Ph), $142.7\left(C\left(5^{\prime}\right)\right), 144.5$ (i-Ph), $171.2(C(1)) ; \mathrm{m} / \mathrm{z}\left(\mathrm{ESI}^{+}\right)$ $406\left([\mathrm{M}+\mathrm{H}]^{+}, 100 \%\right)$; HRMS $\left(\mathrm{ESI}^{+}\right) \mathrm{C}_{26} \mathrm{H}_{32} \mathrm{NO}_{3}{ }^{+}\left([\mathrm{M}+\mathrm{H}]^{+}\right)$requires 406.2377; found 406.2370.

tert-Butyl $\quad(3 S, \alpha R)-3-[N$-(furan-3'-ylmethyl)- $N$-( $\alpha$-methylbenzyl)amino]-3-phenylpropanoate 31 : Method A: $\mathrm{PPh}_{3}(3.48 \mathrm{~g}, 13.3 \mathrm{mmol}$ ) was added to a stirred solution of 3-(hydroxymethyl)furan (0.88 mL, $10.2 \mathrm{mmol})$ and $\mathrm{CBr}_{4}(4.02 \mathrm{~g}, 12.2 \mathrm{mmol})$ in $\mathrm{CH}_{2} \mathrm{Cl}_{2}(51.0 \mathrm{~mL})$ at rt. The resultant mixture was left to stir at $\mathrm{rt}$ for $3 \mathrm{~h}$, before being poured over hexane $(200 \mathrm{~mL})$. The resultant mixture was filtered through Celite $^{\circledR}$ (eluent hexane) then concentrated in vacuo. Secondary amine (3S, $\alpha R$ )-35 (331 mg, $1.02 \mathrm{mmol}$, $>95: 5 \mathrm{dr}$ ) was added to the residue, and the resultant mixture was left to stir at rt for $16 \mathrm{~h}$, then concentrated in vacuo. Purification via flash column chromatography (eluent $30-40{ }^{\circ} \mathrm{C}$ petrol/ $\mathrm{Et}_{2} \mathrm{O} / 35 \%$ aqNH$\left.{ }_{4} \mathrm{OH}, 90: 9: 1\right)$ gave (3S, $\left.\alpha R\right)-31$ as a white solid (114 mg, 28\%, >80\% purity).

Method B: Following General procedure 2, s-BuLi (1.4 M in cyclohexane, $1.17 \mathrm{~mL} 1.52 \mathrm{mmol}$ ) was reacted with (R)-22 (315 mg, $1.57 \mathrm{mmol}$ ) and 24 (200 mg, $0.98 \mathrm{mmol},>95: 5 \mathrm{dr}[(E):(Z)])$ in THF (3.92 $\mathrm{mL}$ ). Purification via flash column chromatography (eluent $30-40{ }^{\circ} \mathrm{C}$ petrol $/ \mathrm{Et}_{2} \mathrm{O} / 35 \%$ aqNH $4 \mathrm{OH}_{\text {, }}$ 90:9:1) gave (3S, $\alpha R)-31$ as a colorless solid (247 mg, 62\%, >95:5 dr); $[\alpha]_{\mathrm{D}}^{22}+3.2$ (c 1.0 in $\left.\mathrm{CHCl}_{3}\right)$; mp 90-92 ${ }^{\circ} \mathrm{C}$.

tert-Butyl (3RS, $\alpha S R)$-3-[N-(furan-2'-ylmethyl)- $N$-( $\alpha$-methylbenzyl)amino]-3-phenylpropanoate 32: Following General procedure 2, s-BuLi (1.4 M in cyclohexane, $1.09 \mathrm{~mL} 1.52 \mathrm{mmol}$ ) was reacted with 23 (315 mg, $1.57 \mathrm{mmol}$ ) and 24 (200 mg, $0.98 \mathrm{mmol},>95: 5 \mathrm{dr}$ [(E):(Z)]) in THF (3.92 mL). Purification via flash column chromatography (eluent $30-40{ }^{\circ} \mathrm{C}$ petrol/ $\mathrm{Et}_{2} \mathrm{O} / 35 \%$ aqNH${ }_{4} \mathrm{OH}, 93: 6: 1$ ) gave 32 as a pale yellow oil (306 mg, 77\%, 62:38 dr): $v_{\max }(\mathrm{ATR}) 1726(\mathrm{C}=\mathrm{O})$; $\delta_{\mathrm{H}}\left(400 \mathrm{MHz}, \mathrm{CDCl}_{3}\right)$ [selected peaks for the major diastereoisomer] $1.24(9 \mathrm{H}, \mathrm{s}, \mathrm{CMe}), 1.27$ (3H, d, $J$ 6.6, $\mathrm{C}(\alpha) M e), 2.52(1 \mathrm{H}, \mathrm{dd}, J$ 14.5, 9.8, C(2) $\left.H_{\mathrm{A}}\right), 2.67\left(1 \mathrm{H}, \mathrm{dd}, J\right.$ 14.5, 5.2, C(2)H $\left.H_{\mathrm{B}}\right), 3.65\left(1 \mathrm{H}, \mathrm{d}, J 15.9, \mathrm{NCH}_{\mathrm{A}} \mathrm{H}_{\mathrm{B}} \mathrm{Ar}\right), 3.70(1 \mathrm{H}, \mathrm{d}, J$ 15.9, $\left.\mathrm{NCH}_{\mathrm{A}} H_{\mathrm{B}} \mathrm{Ar}\right), 4.05(1 \mathrm{H}, \mathrm{q}, J$ 6.6, C( $\left.\alpha) H\right), 4.43(1 \mathrm{H}, \mathrm{dd}, J$ 9.8, 5.2, C(3)H), $6.10(1 \mathrm{H}, \mathrm{dd}, J$ 3.2, 0.9, $\left.\mathrm{C}\left(3^{\prime}\right) H\right), 6.29\left(1 \mathrm{H}, \mathrm{dd}, J 3.2,1.9, \mathrm{C}\left(4^{\prime}\right) H\right) ; \delta_{\mathrm{H}}\left(400 \mathrm{MHz}^{\prime} \mathrm{CDCl}_{3}\right)$ [selected peaks for the minor diastereoisomer] $1.28(9 \mathrm{H}, \mathrm{s}, \mathrm{CMe}$ ), 1.39 (3H, d, J 6.6, C( $\alpha) M e), 2.70$ (1H, dd, J 14.6, 10.1, C(2)H $\left.H_{\mathrm{A}}\right)$, $2.82(1 \mathrm{H}, \mathrm{dd}, J$ 14.6, 4.9, C(2)HB $), 3.51\left(1 \mathrm{H}, \mathrm{d}, J\right.$ 15.9, $\left.\mathrm{NCH}_{\mathrm{A}} \mathrm{H}_{\mathrm{B}} \mathrm{Ar}\right), 3.77\left(1 \mathrm{H}, \mathrm{d}, J\right.$ 15.9, $\left.\mathrm{NCH}_{\mathrm{A}} H_{\mathrm{B}} \mathrm{Ar}\right)$, $4.05(1 \mathrm{H}, \mathrm{q}, J$ 6.6, C( $\alpha) H), 4.51$ (1H, dd, $J$ 10.1, 4.9, C(3)H), $6.06\left(1 \mathrm{H}, \mathrm{dd}, J 3.2,0.9, \mathrm{C}\left(3^{\prime}\right) H\right), 6.27(1 \mathrm{H}$, dd, $J$ 3.2, 1.9, $\left.\mathrm{C}\left(4^{\prime}\right) H\right)$; $\delta_{\mathrm{C}}\left(100 \mathrm{MHz}, \mathrm{CDCl}_{3}\right)$ [selected peaks for the major diastereoisomer] 16.8 (C( $\alpha) M e), 27.8\left(\mathrm{CMe}_{3}\right), 38.5(C(2)), 43.6\left(\mathrm{NCH}_{2} \mathrm{Ar}\right), 57.0(C(\alpha)), 59.5(C(3)), 80.1\left(C \mathrm{Me}_{3}\right), 107.2\left(C\left(3^{\prime}\right)\right)$, $110.3\left(C\left(4^{\prime}\right)\right), 140.8(i-P h), 141.1\left(C\left(5^{\prime}\right)\right), 144.6(i-P h), 154.9\left(C\left(2^{\prime}\right)\right), 171.2(C(1)) ; \delta_{C}\left(100 \mathrm{MHz}, \mathrm{CDCl}_{3}\right)$ [selected peaks for the minor diastereoisomer] $19.4(\mathrm{C}(\alpha) M e), 27.9\left(\mathrm{CMe}_{3}\right), 38.1(C(2)), 43.0\left(\mathrm{NCH}_{2} \mathrm{Ar}\right)$, 
$57.9(C(\alpha)), 59.6(C(3)), 80.3\left(C \mathrm{Me}_{3}\right), 107.4\left(C\left(3^{\prime}\right)\right), 110.2\left(C\left(4^{\prime}\right)\right), 141.1\left(C\left(5^{\prime}\right)\right), 141.4(i-P h), 144.8$

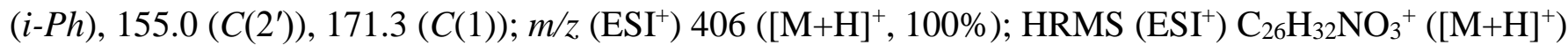
requires 406.2377; found 406.2374.

tert-Butyl (3S, $\alpha R)-3-\left[N\right.$-benzyl- $N$-( $\alpha$-methylbenzy)amino]-3-phenylpropanoate $34:^{11 \mathrm{c}} n$-BuLi $(2.5 \mathrm{M}$ in hexanes, $7.35 \mathrm{~mL}, 18.4 \mathrm{mmol})$ was added dropwise via syringe to a stirred solution of $(R)-33$ (4.00 g, $19.0 \mathrm{mmol})$ in THF $(110 \mathrm{~mL})$ at $-78^{\circ} \mathrm{C}$. After stirring for $30 \mathrm{~min}$, a solution of $24(2.42 \mathrm{~g}, 11.8 \mathrm{mmol}$, $>95: 5 \mathrm{dr}[(E):(Z)])$ in THF $(8 \mathrm{~mL})$ at $-78{ }^{\circ} \mathrm{C}$ was added dropwise via cannula. The reaction mixture was left to stir for $2 \mathrm{~h}$ before the addition of satd aq $\mathrm{NH}_{4} \mathrm{Cl}(10 \mathrm{~mL})$. The resultant mixture was allowed to warm to rt over $15 \mathrm{~min}$, then concentrated in vacuo. The residue was then partitioned between $\mathrm{CH}_{2} \mathrm{Cl}_{2}$ $(100 \mathrm{~mL})$ and $10 \%$ aq citric acid solution $(100 \mathrm{~mL})$. The aqueous layer was extracted with $\mathrm{CH}_{2} \mathrm{Cl}_{2}(2 \times$ $100 \mathrm{~mL}$ ) and the combined organic extracts were washed sequentially with satd aq $\mathrm{NaHCO}_{3}(200 \mathrm{~mL})$, $\mathrm{H}_{2} \mathrm{O}(200 \mathrm{~mL})$ and brine $(200 \mathrm{~mL})$, then dried and concentrated in vacuo. Purification via flash column chromatography (eluent $30-40{ }^{\circ} \mathrm{C}$ petrol/Et $2 \mathrm{O}, 15: 1$ ) gave 34 as a pale yellow oil (4.80 mg, 98\%, >95:5 dr); $[\alpha]_{\mathrm{D}}^{22}+3.6$ (c 1.0 in $\left.\mathrm{CHCl}_{3}\right)$; $\left\{\right.$ lit. ${ }^{11 \mathrm{c}}[\alpha]_{\mathrm{D}}^{22}+3.9\left(\right.$ c 0.7 in $\left.\left.\mathrm{CHCl}_{3}\right)\right\} ; \delta_{\mathrm{H}}\left(400 \mathrm{MHz}, \mathrm{CDCl}_{3}\right) 1.23(9 \mathrm{H}, \mathrm{s}$, $\left.\mathrm{CMe}_{3}\right), 1.27$ (3H, d, $J$ 6.8, C( $\left.\alpha\right) M e$ ), 2.50 (1H, dd, $J$ 14.4, 9.9, C(2)H $\left.H_{\mathrm{A}}\right), 2.56$ (1H, dd, $\left.J 14.4,5.2, \mathrm{C}(2) H_{\mathrm{B}}\right)$,

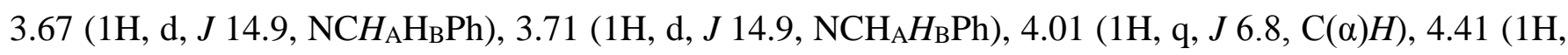
dd, $J$ 9.9, 5.2, C(3)H), 7.17-7.44 (15H, m, Ph).

tert-Butyl (3S, $\alpha R)$-3-phenyl-4-( $N$ - $\alpha$-methylbenzyl)amino-propanoate 35: ${ }^{21}$ CAN (17.6 g, 32.1 mmol) was added to a stirred solution of (3S, $\alpha R)-34$ (6.52 g, $15.7 \mathrm{mmol},>95: 5 \mathrm{dr})$ in $\mathrm{MeCN} / \mathrm{H}_{2} \mathrm{O}$ (v/v 5:1, 184 $\mathrm{mL})$ at rt. The reaction mixture was stirred at rt for $2 \mathrm{~h}$ before the addition of satd aq $\mathrm{NaHCO}_{3}(130 \mathrm{~mL})$. The resultant mixture was stirred at rt for $30 \mathrm{~min}$. The aqueous layer was extracted with ethyl acetate (3 $\times 100 \mathrm{~mL}$ ), and the combined organics were then dried and concentrated in vacuo. The residue was then stirred in $2.0 \mathrm{M}$ aq $\mathrm{NaHSO}_{3}(100 \mathrm{~mL})$ for $20 \mathrm{~min}$. The resultant mixture was extracted with $\mathrm{Et}_{2} \mathrm{O}(3 \times$ $100 \mathrm{~mL}$ ), and the combined organic extracts were dried and concentrated in vacuo. Purification via flash column chromatography (eluent $30-40{ }^{\circ} \mathrm{C}$ petrol/ $\mathrm{Et}_{2} \mathrm{O} / 35 \%$ aqNH $\left.\mathrm{H}_{4} \mathrm{OH}, 80: 19: 1\right)$ gave (3S, $\alpha R$ )-35 as a pale yellow oil (4.42 g, 87\%, >95:5 dr); $[\alpha]_{\mathrm{D}}^{22}+12.6$ (c 1.0 in $\left.\mathrm{CHCl}_{3}\right)$; $\delta_{\mathrm{H}}\left(400 \mathrm{MHz}, \mathrm{CDCl}_{3}\right) 1.36(3 \mathrm{H}, \mathrm{d}$, $J$ 6.5, C( $\alpha) M e), 1.38$ (9H, s, CMe $)$ ), 2.59 (1H, dd, J14.7, 6.2, C(2) $\left.H_{\mathrm{A}}\right), 2.68\left(1 \mathrm{H}, \mathrm{dd}, J 14.7,7.9, \mathrm{C}(2) H_{\mathrm{B}}\right)$, 3.66 (1H, q, $J$ 6.5, C $(\alpha) H)$, 4.16 (1H, dd, J 7.9, 6.2, C(3)H), 7.19-7.33 (10H, m, Ph).

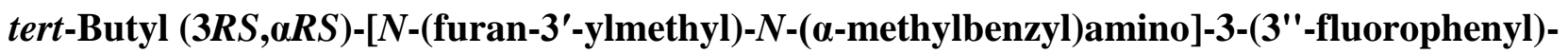

propanoate 41: Following General procedure 2, s-BuLi (1.4 M in cyclohexane, $0.99 \mathrm{~mL}, 1.40 \mathrm{mmol})$ was reacted with 22 (290 mg, $1.44 \mathrm{mmol}$ ) and 37 (200 mg, $0.90 \mathrm{mmol},>95: 5 \mathrm{dr}[(E):(Z)])$ in THF (3.61 $\mathrm{mL}$ ). Purification via flash column chromatography (eluent $30-40{ }^{\circ} \mathrm{C}$ petrol $/ \mathrm{Et}_{2} \mathrm{O} / 35 \%$ aqNH $4 \mathrm{OH}_{\text {, }}$ 90:9:1) gave 41 as a pale yellow oil (325 mg, 85\%, >95:5 dr); $v_{\max }(\mathrm{ATR}) 1724$ (C=O); $\delta_{\mathrm{H}}(400 \mathrm{MHz}$, 
$\left.\mathrm{CDCl}_{3}\right) 1.29$ (9H, s, $\left.\mathrm{CMe}_{3}\right), 1.29$ (3H, d, $J$ 6.8, C( $\left.\left.\alpha\right) M e\right), 2.52$ (1H, dd, $J$ 14.8, 9.8, C(2)H $\left.H_{\mathrm{A}}\right), 2.57$ (1H, dd, $J$ 14.8, 5.0, C(2)Hв), 3.51 (2H, app s, $\left.\mathrm{NCH}_{2} \mathrm{Ar}\right), 4.02$ (1H, q, $J$ 6.8, C( $\left.\left.\alpha\right) H\right), 4.45$ (1H, dd, $J$ 9.8, 5.0, C(3)H), 6.26 (1H, dd, $J$ 1.7, 0.7, C(4')H), 6.96 (1H, tdd, $J$ 8.4, 2.6, 1.0, C(4")H), 7.13-7.42 (10H, m, $\left.\mathrm{C}\left(2^{\prime}\right) H, \mathrm{C}\left(5^{\prime}\right) H, \mathrm{C}\left(2^{\prime \prime}\right) H, \mathrm{C}\left(5^{\prime \prime}\right) H, \mathrm{C}\left(6^{\prime \prime}\right) H, P h\right) ; \delta_{\mathrm{C}}\left(100 \mathrm{MHz}, \mathrm{CDCl}_{3}\right) 16.8(\mathrm{C}(\alpha) M e), 27.8\left(\mathrm{CMe}_{3}\right), 38.1$ (C(2)), $41.6\left(\mathrm{NCH}_{2} \mathrm{Ar}\right), 56.8(C(\alpha)), 58.6(C(3))$, $80.4\left(\mathrm{CMe}_{3}\right), 110.7\left(C\left(4^{\prime}\right)\right), 113.9\left(\mathrm{~d}, J\right.$ 21.0, $\left.C\left(4^{\prime \prime}\right)\right)$, 115.0 (d, $J$ 21.9, $\left.C\left(2^{\prime \prime}\right)\right), 123.6$ (d, $J$ 2.9, $\left.C\left(6^{\prime \prime}\right)\right), 125.0$ (C(3')), 126.9, 127.6, 128.2 (o,m,p-Ph), 129.5 (d, $J$ 8.6, $\left.C\left(5^{\prime \prime}\right)\right), 140.0\left(C\left(2^{\prime}\right)\right), 142.9\left(C\left(5^{\prime}\right)\right), 144.0(i-P h), 145.0\left(\mathrm{~d}, J\right.$ 5.7, $\left.C\left(1^{\prime \prime}\right)\right), 162.8\left(\mathrm{~d}, J\right.$ 245.1, $\left.C\left(3^{\prime \prime}\right)\right)$, $170.9(C(1)) ; \delta_{\mathrm{F}}\left(377 \mathrm{MHz}, \mathrm{CDCl}_{3}\right)-113.6\left(\mathrm{C}\left(3^{\prime \prime}\right) \mathrm{F}\right) ; \mathrm{m} / \mathrm{z}\left(\mathrm{ESI}^{+}\right) 424\left([\mathrm{M}+\mathrm{H}]^{+}, 100 \%\right) ; \mathrm{HRMS}\left(\mathrm{ESI}^{+}\right)$ $\mathrm{C}_{26} \mathrm{H}_{31} \mathrm{FNO}_{3}{ }^{+}\left([\mathrm{M}+\mathrm{H}]^{+}\right)$requires 424.2282; found 424.2277 .

\section{tert-Butyl (3RS,aRS)-3-[N-(furan-3'-ylmethyl)- $N$-( $\alpha$-methylbenzyl)amino]-3-(3"'-methoxyphenyl)-}

propanoate 42: Following General procedure 2, s-BuLi (1.4 M in cyclohexane, $0.95 \mathrm{~mL}, 1.33 \mathrm{mmol})$ was reacted with 22 (275 mg, $1.37 \mathrm{mmol}$ ) and 38 (200 mg, $0.86 \mathrm{mmol},>95: 5 \mathrm{dr}[(E):(Z)])$ in THF (3.42 $\mathrm{mL}$ ). Purification via flash column chromatography (eluent $30-40{ }^{\circ} \mathrm{C}$ petrol/Et $2 \mathrm{O} / 35 \%$ aqNH $\mathrm{OH}_{4}$, 90:9:1) gave 42 as a pale yellow oil (257 mg, 69\%, >95:5 dr); $v_{\max }$ (ATR) 1724 (C=O); $\delta_{\mathrm{H}}(500 \mathrm{MHz}$, $\left.\mathrm{CDCl}_{3}\right) 1.27$ (3H, d, $J$ 6.8, C( $\left.\left.\alpha\right) M e\right), 1.29$ (9H, s, CMe $\left.e_{3}\right), 2.54$ (1H, dd, $J$ 14.8, 9.6, C(2) $\left.H_{\mathrm{A}}\right), 2.61(1 \mathrm{H}, \mathrm{dd}$,

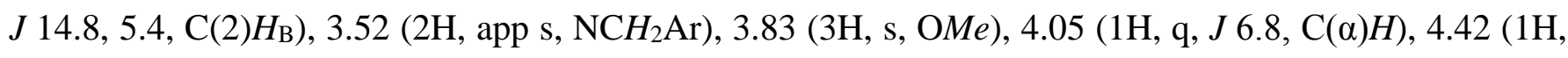
dd, J 9.6, 5.4, C(3)H), $6.17\left(1 \mathrm{H}, \mathrm{dd}, J 1.7,0.7, \mathrm{C}\left(4^{\prime}\right) H\right), 6.79-7.43\left(11 \mathrm{H}, \mathrm{m}, \mathrm{C}\left(2^{\prime}\right) H, \mathrm{C}\left(5^{\prime}\right) H, \mathrm{C}\left(2^{\prime \prime}\right) H\right.$, $\left.\mathrm{C}\left(3^{\prime \prime}\right) H, \mathrm{C}\left(5^{\prime \prime}\right) H, \mathrm{C}\left(6^{\prime \prime}\right) H, P h\right) ; \delta_{\mathrm{C}}\left(125 \mathrm{MHz}, \mathrm{CDCl}_{3}\right) 16.4(\mathrm{C}(\alpha) M e), 27.8\left(\mathrm{CMe}_{3}\right), 38.7(C(2)), 41.6$ $\left(\mathrm{NCH}_{2} \mathrm{Ar}\right), 55.2(\mathrm{OMe}), 56.6(C(\alpha)), 59.1(C(3)), 80.2\left(C \mathrm{Me}_{3}\right), 110.8\left(C\left(4^{\prime}\right)\right), 112.3\left(C\left(4^{\prime \prime}\right)\right), 114.0\left(C\left(2^{\prime \prime}\right)\right)$, $120.4\left(C\left(6^{\prime \prime}\right)\right), 125.3\left(C\left(3^{\prime}\right)\right), 126.7,127.6,128.1$ (o,m,p-Ph), $129.0\left(C\left(5^{\prime \prime}\right)\right), 140.0\left(C\left(2^{\prime}\right)\right), 142.7\left(C\left(5^{\prime}\right)\right)$, $143.6\left(C\left(1^{\prime \prime}\right)\right), 144.4(\mathrm{i}-\mathrm{Ph}), 159.5\left(C\left(3^{\prime \prime}\right)\right), 171.0(C(1)) ; \mathrm{m} / \mathrm{z}\left(\mathrm{ESI}^{+}\right) 436\left([\mathrm{M}+\mathrm{H}]^{+}, 100 \%\right) ; \mathrm{HRMS}\left(\mathrm{ESI}^{+}\right)$ $\mathrm{C}_{27} \mathrm{H}_{34} \mathrm{NO}_{4}{ }^{+}\left([\mathrm{M}+\mathrm{H}]^{+}\right)$requires 436.2482; found 436.2475.

\section{tert-Butyl (3RS, $\alpha R S)-3$-[ $N$-(furan-3'-ylmethyl)- $N$-( $\alpha$-methylbenzyl)amino]-3-(4' '-fluorophenyl)-}

propanoate 43: Following General procedure 2, s-BuLi (1.4 M in cyclohexane, $0.99 \mathrm{~mL}, 1.40 \mathrm{mmol}$ ) was reacted with 23 (290 mg, $1.44 \mathrm{mmol}$ ) and 39 (200 mg, $0.90 \mathrm{mmol}$, >95:5 dr [(E):(Z)]) in THF (3.61 $\mathrm{mL}$ ). Purification via flash column chromatography (eluent $30-40{ }^{\circ} \mathrm{C}$ petrol/Et $2 \mathrm{O} / 35 \%$ aqNH $\mathrm{OH}_{4}$, 90:9:1) gave 43 as a pale yellow oil (186 mg, 49\%, >95:5 dr); $v_{\max }$ (ATR) 1724 (C=O); $\delta_{\mathrm{H}}(500 \mathrm{MHz}$, $\left.\mathrm{CDCl}_{3}\right) 1.26\left(9 \mathrm{H}, \mathrm{s}, \mathrm{CMe} e_{3}\right), 1.27$ (3H, d, $\left.J 6.7, \mathrm{C}(\alpha) M e\right), 2.51\left(1 \mathrm{H}, \mathrm{dd}, J 14.6,10.1, \mathrm{C}(2) H_{\mathrm{A}}\right), 2.60(1 \mathrm{H}, \mathrm{dd}$, $J$ 14.6, 4.8, C(2)HB $) 3.50$ (2H, app s, $\left.\mathrm{NCH}_{2} \mathrm{Ar}\right), 4.00(1 \mathrm{H}, \mathrm{q}, J$ 6.7, $\mathrm{C}(\alpha) H), 4.41(1 \mathrm{H}, \mathrm{dd}, J$ 10.1, 4.8,

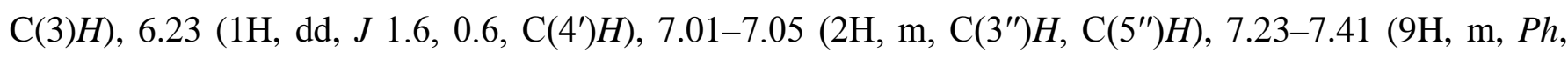
$\left.\mathrm{C}\left(2^{\prime}\right) H, \mathrm{C}\left(5^{\prime}\right) H, \mathrm{C}\left(2^{\prime \prime}\right) H, \mathrm{C}\left(6^{\prime \prime}\right) H\right)$; $\delta_{\mathrm{C}}\left(125 \mathrm{MHz}, \mathrm{CDCl}_{3}\right) 16.4(\mathrm{C}(\alpha) M e), 27.8\left(\mathrm{CMe}_{3}\right), 38.5(C(2)), 41.5$ $\left(\mathrm{NCH}_{2} \mathrm{Ar}\right), 56.7(C(\alpha)), 58.7(C(3)), 80.3\left(C \mathrm{Me}_{3}\right), 110.7\left(C\left(4^{\prime}\right)\right), 114.9\left(\mathrm{~d}, J\right.$ 21.0, $\left.C\left(3^{\prime \prime}\right), C\left(5^{\prime \prime}\right)\right), 125.3$ $\left(C\left(3^{\prime}\right)\right)$, 126.8, 127.6, 128.2 (o,m,p-Ph), 129.6 (d, J 7.6, C(2"), C(6"')), 137.7 (d, J 3.8, C(1")), 139.9 $\left(C\left(2^{\prime}\right)\right), 142.8\left(C\left(5^{\prime}\right)\right), 144.2(i-P h), 161.9\left(\mathrm{~d}, J\right.$ 245.1, $\left.C\left(4^{\prime \prime}\right)\right), 171.0(C(1)) ; \delta_{\mathrm{F}}\left(377 \mathrm{MHz}, \mathrm{CDCl}_{3}\right)-115.8$ 
$\left(\mathrm{C}\left(4^{\prime \prime}\right) \mathrm{F}\right) ; \mathrm{m} / \mathrm{z}\left(\mathrm{ESI}^{+}\right) 424\left([\mathrm{M}+\mathrm{H}]^{+}, 100 \%\right) ; \mathrm{HRMS}\left(\mathrm{ESI}^{+}\right) \mathrm{C}_{26} \mathrm{H}_{31} \mathrm{FNO}_{3}{ }^{+}\left([\mathrm{M}+\mathrm{H}]^{+}\right)$requires 424.2282; found 424.2279 .

\section{tert-Butyl (3RS,aRS)-3-[N-(furan-3'-ylmethyl)- $N$-( $\alpha$-methylbenzyl)amino]-3-(4' '-methoxyphenyl)-}

propanoate 44: Following General procedure 2, s-BuLi (1.4 M in cyclohexane, $0.95 \mathrm{~mL}, 1.33 \mathrm{mmol})$ was reacted with 22 (275 mg, $1.37 \mathrm{mmol})$ and 40 (200 mg, $0.86 \mathrm{mmol},>95: 5 \mathrm{dr}[(E):(Z)])$ in THF (3.42 $\mathrm{mL}$ ). Purification via flash column chromatography (eluent $30-40{ }^{\circ} \mathrm{C}$ petrol $/ \mathrm{Et}_{2} \mathrm{O} / 35 \%$ aqNH $4 \mathrm{OH}_{\text {, }}$ 90:9:1) gave 44 as a pale yellow oil (195 mg, 52\%, >95:5 dr); $v_{\max }$ (ATR) 1725 (C=O); $\delta_{\mathrm{H}}(500 \mathrm{MHz}$, $\left.\mathrm{CDCl}_{3}\right) 1.25$ (3H, d, $J$ 6.8, C( $\left.\left.\alpha\right) M e\right), 1.27$ (9H, s, CMe3), 2.52 (1H, dd, $J$ 14.5, 10.1, C(2)H $\left.H_{\mathrm{A}}\right), 2.61(1 \mathrm{H}, \mathrm{dd}$, $J$ 14.5, 5.0, C(2)Hв), 3.50 (2H, app s, $\left.\mathrm{NCH}_{2} \mathrm{Ar}\right), 3.81$ (3H, s, OMe), 4.02 (1H, q, $J$ 6.8, C( $\left.\left.\alpha\right) H\right), 4.38(1 \mathrm{H}$, dd, $J$ 10.1, 5.0, C(3)H), 6.24 (1H, dd, $J$ 1.7, 0.7, C(4')H), 6.88 (2H, d, $J$ 8.5, C(3")H, C(5')H), 7.21-7.43 $\left(9 \mathrm{H}, \mathrm{m}, \mathrm{C}\left(2^{\prime}\right) H, \mathrm{C}\left(5^{\prime}\right) H, \mathrm{C}\left(2^{\prime \prime}\right) H, \mathrm{C}\left(6^{\prime \prime}\right) H, P h\right) ; \delta_{\mathrm{C}}\left(125 \mathrm{MHz}, \mathrm{CDCl}_{3}\right) 16.2(\mathrm{C}(\alpha) M e), 27.8(\mathrm{CMe})$ ), 38.9 (C(2)), $41.4\left(\mathrm{NCH}_{2} \mathrm{Ar}\right), 55.2(\mathrm{OMe}), 56.5(C(\alpha)), 58.7$ (C(3)), $80.1\left(C \mathrm{Me}_{3}\right), 110.8\left(C\left(4^{\prime}\right)\right), 113.4\left(C\left(3^{\prime \prime}\right)\right.$, $\left.C\left(5^{\prime \prime}\right)\right), 125.5\left(C\left(3^{\prime}\right)\right)$, 126.7, 127.6, $128.0\left(\right.$ o,m,p-Ph), 129.2 (C(2'), $\left.C\left(6^{\prime \prime}\right)\right), 133.8\left(C\left(1^{\prime \prime}\right)\right), 139.9\left(C\left(2^{\prime}\right)\right)$, $142.6\left(C\left(5^{\prime}\right)\right), 144.6(\mathrm{i}-\mathrm{Ph}), 158.6\left(C\left(4^{\prime \prime}\right)\right), 171.2(C(1)) ; \mathrm{m} / \mathrm{z}\left(\mathrm{ESI}^{+}\right) 436\left([\mathrm{M}+\mathrm{H}]^{+}, 100 \%\right) ; \mathrm{HRMS}\left(\mathrm{ESI}^{+}\right)$ $\mathrm{C}_{27} \mathrm{H}_{34} \mathrm{NO}_{4}{ }^{+}\left([\mathrm{M}+\mathrm{H}]^{+}\right)$requires 436.2482; found 436.2479 .

\section{tert-Butyl (RS,RS,RS)-2-hydroxy-3-[ $N$-(thiophen-3'-ylmethyl)- $N$-( $\alpha$-methylbenzyl)amino]-3-phenyl-}

propanoate 45: Following General procedure 3, s-BuLi (1.4 M in cyclohexane, $1.85 \mathrm{~mL}, 2.41 \mathrm{mmol}$ ) was reacted with 20 (540 mg, $2.49 \mathrm{mmol}$ ) and 24 (317 mg, $1.56 \mathrm{mmol}$, >95:5 dr [(E):(Z)]) in THF (6.22 $\mathrm{mL}$ ) before the addition of $\mathbf{1 4}$ (605 $\mathrm{mg}, 2.64 \mathrm{mmol}$ ). Purification via flash column chromatography (eluent $30-40{ }^{\circ} \mathrm{C}$ petrol/ $/ \mathrm{Et}_{2} \mathrm{O} / 35 \%$ aqNH $4 \mathrm{OH}, 90: 9: 1$ ) gave 45 as a pale yellow oil (394 mg, 58\%, >95:5 dr); $v_{\max }(\mathrm{ATR}) 3493(\mathrm{O}-\mathrm{H}), 1724(\mathrm{C}=\mathrm{O}) ; \delta_{\mathrm{H}}\left(400 \mathrm{MHz}, \mathrm{CDCl}_{3}\right) 1.21$ (3H, d, J 6.8, C( $\left.\left.\alpha\right) M e\right), 1.22$ (9H, s, CMe 3 ), 3.84 (1H, d, $J$ 15.1, $\left.\mathrm{NCH}_{\mathrm{A}} \mathrm{H}_{\mathrm{B}} \mathrm{Ar}\right), 4.03$ (1H, d, $J$ 15.1, $\left.\mathrm{NCH}_{\mathrm{A}} H_{\mathrm{B}} \mathrm{Ar}\right), 4.21(1 \mathrm{H}, \mathrm{q}, J$ 6.8, C( $\alpha) H$ ), 4.21 (1H, d, $J$ 3.2, C(3)H), 4.42 (1H, d, $J$ 3.2, C(2)H), 6.94 (1H, dd, $J$ 4.9, 1.0, C(4')H), 7.04 (1H, dd, $J$ 2.9, 1.0, C(2')H), 7.17-7.49 (11H, m, C(5')H, Ph); $\delta_{\mathrm{C}}\left(100 \mathrm{MHz}, \mathrm{CDCl}_{3}\right) 13.7(\mathrm{C}(\alpha) M e), 27.7\left(\mathrm{CMe}_{3}\right)$, $47.4\left(\mathrm{NCH}_{2} \mathrm{Ar}\right), 57.0(C(\alpha)), 65.9(C(3)), 73.3(C(2)), 82.2\left(C \mathrm{Me}_{3}\right), 121.4\left(C\left(2^{\prime}\right)\right), 125.5\left(C\left(5^{\prime}\right)\right), 126.8$, 127.6, 127.8, 128.0, 128.1, 129.8 (C(4'), o,m,p-Ph), 138.1 (i-Ph), $143.0\left(C\left(3^{\prime}\right)\right), 144.1$ (i-Ph), 171.9 $(C(1)) ; \mathrm{m} / \mathrm{z}\left(\mathrm{ESI}^{+}\right) 438\left([\mathrm{M}+\mathrm{H}]^{+}, 100 \%\right) ; \mathrm{HRMS}\left(\mathrm{ESI}^{+}\right) \mathrm{C}_{26} \mathrm{H}_{32} \mathrm{NO}_{3} \mathrm{~S}^{+}\left([\mathrm{M}+\mathrm{H}]^{+}\right)$requires 438.2097; found 438.2096.

\section{tert-Butyl (RS,RS,RS)-2-hydroxy-3-[ $N$-(thiophen-2'-ylmethyl)- $N$-( $\alpha$-methylbenzyl)amino]-3-phenyl-}

propanoate 46: Following General procedure 3, s-BuLi (1.4 M in cyclohexane, $1.51 \mathrm{~mL}, 1.97 \mathrm{mmol})$ was reacted with 21 (440 mg, $2.03 \mathrm{mmol}$ ) and 24 (259 mg, $1.27 \mathrm{mmol}$, >95:5 dr [(E):(Z)]) in THF (5.07 $\mathrm{mL}$ ) before the addition of $\mathbf{1 4}$ (494 $\mathrm{mg}, 2.16 \mathrm{mmol}$ ). Purification via flash column chromatography (eluent $30-40{ }^{\circ} \mathrm{C}$ petrol/ $\mathrm{Et}_{2} \mathrm{O} / 35 \%$ aqNH$\left._{4} \mathrm{OH}, 90: 9: 1\right)$ gave 46 as a pale yellow oil (267 mg, 48\%, 94:6

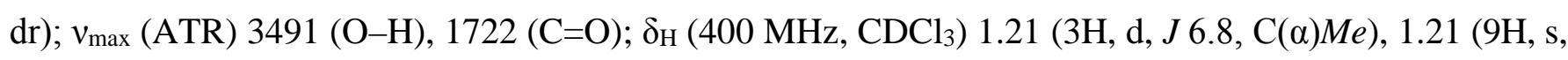


CMe 3 ), 4.11 (1H, d, $J$ 15.4, $\left.\mathrm{NCH}_{\mathrm{A}} \mathrm{H}_{\mathrm{B}} \mathrm{Ar}\right), 4.27$ (1H, d, $J$ 15.4, $\mathrm{NCH}_{\mathrm{A}} H_{\mathrm{B}} \mathrm{Ar}$ ), 4.27 (1H, d, $J$ 2.9, C(3)H), 4.27 (1H, q, $J$ 6.8, C( $\alpha) H), 4.47$ (1H, d, $J$ 2.9, C(2)H), 6.88-6.91 (2H, m, C(3')H, C(4')H), $7.18(1 \mathrm{H}, \mathrm{dd}, J$ 4.8, 1.6, C $\left.\left(5^{\prime}\right) H\right), 7.24-7.55(10 \mathrm{H}, \mathrm{m}, \mathrm{Ph}) ; \delta_{\mathrm{C}}\left(100 \mathrm{MHz}, \mathrm{CDCl}_{3}\right) 14.4(\mathrm{C}(\alpha) \mathrm{Me}), 27.7\left(\mathrm{CMe}_{3}\right), 47.3$ $\left(\mathrm{NCH}_{2} \mathrm{Ar}\right), 57.1(C(\alpha)), 64.6(C(3)), 73.6(C(2))$, $82.3\left(C \mathrm{Me}_{3}\right), 124.4\left(C\left(3^{\prime}\right), C\left(5^{\prime}\right)\right), 126.4\left(C\left(4^{\prime}\right)\right), 126.9$, 127.6, 128.0, 128.2, 129.8 (o,m,p-Ph), 138.1, 143.8 (i-Ph), 146.8 (C(2')), 172.1 (C(1)); m/z (ESI 460 $\left([\mathrm{M}+\mathrm{Na}]^{+}, 100 \%\right) ; \mathrm{HRMS}\left(\mathrm{ESI}^{+}\right) \mathrm{C}_{26} \mathrm{H}_{32} \mathrm{NO}_{3} \mathrm{~S}^{+}\left([\mathrm{M}+\mathrm{H}]^{+}\right)$requires 438.2097; found 438.2094.

tert-Butyl (RS,RS,RS)-2-hydroxy-3-[N-(furan-3'-ylmethyl)- $N$-( $\alpha$-methylbenzyl)amino]-3-phenyl-

propanoate 47: Following General procedure 3, s-BuLi (1.4 M in cyclohexane, $5.43 \mathrm{~mL}, 7.60 \mathrm{mmol}$ ) was reacted with 22 (1.58 g, $7.84 \mathrm{mmol})$ and 24 (1.00 g, $4.90 \mathrm{mmol},>95: 5 \mathrm{dr}[(E):(Z)])$ in THF (19.6 mL) before the addition of 14 (1.91 g, $8.33 \mathrm{mmol}$ ). Purification via flash column chromatography (eluent 30-40 ${ }^{\circ} \mathrm{C}$ petrol/ $/ \mathrm{Et}_{2} \mathrm{O} / 35 \%$ aqNH$\left.{ }_{4} \mathrm{OH}, 90: 9: 1\right)$ gave 47 as a pale yellow oil (1.06 g, 51\%, >95:5 dr); $v_{\max }$ (ATR) $3497(\mathrm{O}-\mathrm{H}), 1724(\mathrm{C}=\mathrm{O}) ; \delta_{\mathrm{H}}\left(500 \mathrm{MHz}, \mathrm{CDCl}_{3}\right) 1.20(3 \mathrm{H}, \mathrm{d}, J 6.8, \mathrm{C}(\alpha) M e), 1.23(9 \mathrm{H}, \mathrm{s}, \mathrm{CMe} 3)$, 3.67 (1H, d, $J$ 15.2, $\left.\mathrm{NCH}_{\mathrm{A}} \mathrm{H}_{\mathrm{B}} \mathrm{Ar}\right), 3.87$ (1H, d, $J$ 15.2, $\left.\mathrm{NCH}_{\mathrm{A}} H_{\mathrm{B}} \mathrm{Ar}\right), 4.21(1 \mathrm{H}, \mathrm{q}, J$ 6.8, C( $\left.\alpha) H\right), 4.23(1 \mathrm{H}$, d, J 3.5, C(3)H), 4.45 (1H, d, J 3.5, C(2)H), 6.21 (1H, dd, J 1.8, 0.9, C(4')H), 7.22-7.49 (12H, m, C(2')H, $\left.\mathrm{C}\left(5^{\prime}\right) H, P h\right) ; \delta_{\mathrm{C}}\left(125 \mathrm{MHz}, \mathrm{CDCl}_{3}\right) 13.5(\mathrm{C}(\alpha) M e), 27.7\left(\mathrm{CMe}_{3}\right), 42.6\left(\mathrm{NCH}_{2} \mathrm{Ar}\right), 56.7(C(\alpha)), 65.9(C(3))$, $73.2(C(2)), 82.2\left(C \mathrm{Me}_{3}\right), 110.6\left(C\left(4^{\prime}\right)\right), 126.8\left(C\left(3^{\prime}\right)\right)$, 127.6, 127.9, 128.0, 128.1, 129.7 (o,m,p-Ph), 138.0 (i-Ph), $139.9\left(C\left(2^{\prime}\right)\right), 143.0\left(C\left(5^{\prime}\right)\right), 144.1(i-P h), 171.9(C(1)) ; m / z\left(\mathrm{ESI}^{+}\right) 422\left([\mathrm{M}+\mathrm{H}]^{+}, 100 \%\right)$; HRS $\left(\mathrm{ESI}^{+}\right) \mathrm{C}_{26} \mathrm{H}_{32} \mathrm{NO}_{4}{ }^{+}\left([\mathrm{M}+\mathrm{H}]^{+}\right)$requires 422.2326; found 422.2320.

tert-Butyl $(R, R, R)$-2-hydroxy-3-[ $N$-(furan-3'-ylmethyl)- $N$-( $\alpha$-methylbenzyl)amino]-3-phenyl-

propanoate 47: Following General procedure 3, s-BuLi (1.4 M in cyclohexane, $1.17 \mathrm{~mL}, 1.52 \mathrm{mmol}$ ) was reacted with $(R)-22(315 \mathrm{mg}, 1.57 \mathrm{mmol})$ and 24 (200 mg, $0.98 \mathrm{mmol},>95: 5 \mathrm{dr}[(E):(Z)])$ in THF (3.92 $\mathrm{mL}$ ) before the addition of 14 (382 $\mathrm{mg}, 1.67 \mathrm{mmol}$ ). Purification via flash column chromatography (eluent $30-40{ }^{\circ} \mathrm{C}$ petrol/ $\mathrm{Et}_{2} \mathrm{O} / 35 \%$ aqNH $\left.\mathrm{H}_{4} \mathrm{OH}, 90: 9: 1\right)$ gave $(R, R, R)-47$ as a pale yellow oil (206 $\mathrm{mg}, 50 \%$, $>95: 5 \mathrm{dr}) ;[\alpha]_{\mathrm{D}}^{22}-7.7$ (c 1.0 in $\left.\mathrm{CHCl}_{3}\right)$.

tert-Butyl (RS,RS,RS)-2-hydroxy-3-[N-(furan-3'-ylmethyl)- $N$-( $\alpha$-methylbenzyl)amino]-3-(3"'-fluorophenyl)propanoate 48: Following General procedure 3, s-BuLi (1.4 M in cyclohexane, $1.62 \mathrm{~mL}, 2.27$ mmol) was reacted with 22 (471 mg, $2.34 \mathrm{mmol}$ ) and 37 (325 mg, $1.46 \mathrm{mmol}$, >95:5 dr [(E):(Z)]) in THF (5.86 mL) before the addition of 14 (570 mg, $2.49 \mathrm{mmol}$ ). Purification via flash column chromatography (eluent $30-40{ }^{\circ} \mathrm{C}$ petrol/ $\mathrm{Et}_{2} \mathrm{O} / 35 \%$ aqNH$\left.{ }_{4} \mathrm{OH}, 90: 9: 1\right)$ gave $\mathbf{4 8}$ as a pale yellow oil (296 mg, 46\%, >95:5 dr); $v_{\max }(\mathrm{ATR}) 3635(\mathrm{O}-\mathrm{H}), 1724(\mathrm{C}=\mathrm{O}) ; \delta_{\mathrm{H}}\left(500 \mathrm{MHz}, \mathrm{CDCl}_{3}\right) 1.22(3 \mathrm{H}, \mathrm{d}, \mathrm{J}$ 6.9, C( $\left.\alpha) M e\right), 1.26(9 \mathrm{H}, \mathrm{s}$, CMe $\left.e_{3}\right), 3.71\left(1 \mathrm{H}, \mathrm{d}, J\right.$ 15.2, $\left.\mathrm{NCH}_{\mathrm{A}} \mathrm{H}_{\mathrm{B}} \mathrm{Ar}\right), 3.89\left(1 \mathrm{H}, \mathrm{dd}, J\right.$ 15.2, 0.8, $\left.\mathrm{NCH}_{\mathrm{A}} H_{\mathrm{B}} \mathrm{Ar}\right), 4.22$ (1H, q, $J$ 6.9, $\mathrm{C}(\alpha) H), 4.24(1 \mathrm{H}, \mathrm{d}, J$ 3.0, C(3)H), $4.43(1 \mathrm{H}, \mathrm{d}, J 3.0, \mathrm{C}(2) H), 6.26(1 \mathrm{H}, \mathrm{d}, J$ 0.9, C(4')H), 6.87-7.38 $\left(11 \mathrm{H}, \mathrm{m}, \mathrm{C}\left(2^{\prime}\right) H, \mathrm{C}\left(5^{\prime}\right) H, \mathrm{C}\left(2^{\prime \prime}\right) H, \mathrm{C}\left(4^{\prime \prime}\right) H, \mathrm{C}\left(5^{\prime \prime}\right) H, \mathrm{C}\left(6^{\prime \prime}\right) \mathrm{H}, \mathrm{Ph}\right) ; \delta_{\mathrm{C}}\left(125 \mathrm{MHz}, \mathrm{CDCl}_{3}\right) 14.0(\mathrm{C}(\alpha) M e)$, 
$27.7\left(\mathrm{CMe}_{3}\right), 42.6\left(\mathrm{NCH}_{2} \mathrm{Ar}\right), 56.8(C(\alpha)), 64.8$ (C(3)), $73.1(C(2)), 82.5\left(C \mathrm{Me}_{3}\right), 110.6\left(C\left(4^{\prime}\right)\right), 114.5(\mathrm{~d}$, $J$ 21.0, C(4")), $116.6\left(\mathrm{~d}, J\right.$ 21.9, C(2")), $125.0\left(C\left(3^{\prime}\right)\right), 125.3\left(\mathrm{~d}, J\right.$ 2.9, $\left.C\left(6^{\prime \prime}\right)\right), 126.9,127.8,128.2$ (o,m,p-Ph), 129.4 (d, J 7.6, C(5')), $140.0\left(C\left(2^{\prime}\right)\right), 140.9$ (d, J 6.7, C(1"')), $143.0\left(C\left(5^{\prime}\right)\right), 143.8(i-P h)$, 162.5 (d, $J$ 245.1, C(3")), $171.9(C(1)) ; \delta_{\mathrm{F}}\left(377 \mathrm{MHz}, \mathrm{CDCl}_{3}\right)-113.3\left(\mathrm{C}\left(3^{\prime \prime}\right) F\right) ; \mathrm{m} / \mathrm{z}\left(\mathrm{ESI}^{+}\right) 462\left([\mathrm{M}+\mathrm{Na}]^{+}\right.$, 100\%); HRMS $\left(\mathrm{ESI}^{+}\right) \mathrm{C}_{26} \mathrm{H}_{31} \mathrm{FNO}_{4}{ }^{+}\left([\mathrm{M}+\mathrm{H}]^{+}\right)$requires 440.2232; found 440.2227.

tert-Butyl (RS,RS,RS)-2-hydroxy-3-[N-(furan-3'-ylmethyl)- $N$-( $\alpha$-methylbenzyl)amino]-3-

(3"-methoxyphenyl)propanoate 49: Following General procedure 3, s-BuLi (1.4 M in cyclohexane, $1.54 \mathrm{~mL}, 2.15 \mathrm{mmol}$ ) was reacted with 22 (447 mg, $2.22 \mathrm{mmol}$ ) and 38 (325 mg, $1.39 \mathrm{mmol}$, >95:5 dr [(E):(Z)]) in THF (5.56 mL) before the addition of 14 (541 mg, $2.36 \mathrm{mmol})$. Purification via flash column chromatography (eluent $30-40{ }^{\circ} \mathrm{C}$ petrol/ $\mathrm{Et}_{2} \mathrm{O} / 35 \%$ aqNH $\mathrm{H}_{4} \mathrm{OH}, 90: 9: 1$ ) gave $\mathbf{4 9}$ as a pale yellow oil (288 mg, 46\%, >95:5 dr); $v_{\max }(\mathrm{ATR}) 3635(\mathrm{O}-\mathrm{H}), 1724(\mathrm{C}=\mathrm{O}) ; \delta_{\mathrm{H}}\left(500 \mathrm{MHz}, \mathrm{CDCl}_{3}\right) 1.22$ (3H, d, J 6.8, $\mathrm{C}(\alpha) M e), 1.26$ (9H, s, CMe $)$ ), 3.69 (1H, d, $J$ 15.2, $\mathrm{NCH}_{\mathrm{A}} \mathrm{H}_{\mathrm{B}} \mathrm{Ar}$ ), 3.80 (3H, s, OMe), 3.87 (1H, dd, $J$ 15.2, 1.0, $\left.\mathrm{NCH}_{\mathrm{A}} H_{\mathrm{B}} \mathrm{Ar}\right), 4.21$ (1H, d, $J$ 3.2, C(3)H), $4.23(1 \mathrm{H}, \mathrm{q}, J$ 6.8, C( $\left.\alpha) H\right), 4.42(1 \mathrm{H}, \mathrm{d}, J 3.2, \mathrm{C}(2) H), 6.25$ (1H, dd, $J$ 1.7, 1.0, C(4')H), 6.82-7.48 (11H, m, C $\left.\left(2^{\prime}\right) H, \mathrm{C}\left(5^{\prime}\right) H, \mathrm{C}\left(2^{\prime \prime}\right) H, \mathrm{C}\left(4^{\prime \prime}\right) H, \mathrm{C}\left(5^{\prime \prime}\right) H, \mathrm{C}\left(6^{\prime \prime}\right) H, P h\right)$; $\delta_{\mathrm{C}}\left(125 \mathrm{MHz}, \mathrm{CDCl}_{3}\right) 13.8(\mathrm{C}(\alpha) M e), 27.7\left(\mathrm{CMe}_{3}\right), 42.6\left(\mathrm{NCH}_{2} \mathrm{Ar}\right), 55.2(\mathrm{OMe}), 56.7(C(\alpha)), 65.5(C(3))$, $73.2(C(2)), 80.6\left(C \mathrm{Me}_{3}\right), 110.7\left(C\left(4^{\prime}\right)\right), 112.8\left(C\left(4^{\prime \prime}\right)\right), 115.6\left(C\left(2^{\prime \prime}\right)\right), 122.1\left(C\left(6^{\prime \prime}\right)\right), 125.3\left(C\left(3^{\prime}\right)\right), 126.8$,

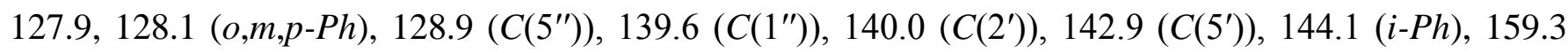
$\left(C\left(3^{\prime \prime}\right)\right), 172.0(C(1)) ; \mathrm{m} / \mathrm{z}\left(\mathrm{ESI}^{+}\right) 452\left([\mathrm{M}+\mathrm{H}]^{+}, 100 \%\right)$; HRMS $\left(\mathrm{ESI}^{+}\right) \mathrm{C}_{27} \mathrm{H}_{34} \mathrm{NO}_{5}{ }^{+}\left([\mathrm{M}+\mathrm{H}]^{+}\right)$requires 452.2431; found 452.2427 .

tert-Butyl (RS,RS,RS)-2-hydroxy-3-[ $N$-(furan-3'-ylmethyl)- $N$-( $\alpha$-methylbenzyl)amino]-3-(4"'-fluorophenyl)propanoate 50: Following General procedure 3, s-BuLi (1.4 M in cyclohexane, $1.50 \mathrm{~mL}, 2.10$ mmol) was reacted with 22 (435 mg, $2.16 \mathrm{mmol}$ ) and 39 (300 mg, $1.35 \mathrm{mmol}$, >95:5 dr [(E):(Z)]) in THF (5.41 mL) before the addition of 14 (526 mg, $2.30 \mathrm{mmol})$. Purification via flash column chromatography (eluent $30-40{ }^{\circ} \mathrm{C}$ petrol/ $\mathrm{Et}_{2} \mathrm{O} / 35 \%$ aqNH$\left.{ }_{4} \mathrm{OH}, 90: 9: 1\right)$ gave 50 as a pale yellow oil (225 mg, 38\%, 94:6 dr); $v_{\max }(\mathrm{ATR}) 3635(\mathrm{O}-\mathrm{H}), 1724(\mathrm{C}=\mathrm{O}) ; \delta_{\mathrm{H}}\left(500 \mathrm{MHz}, \mathrm{CDCl}_{3}\right) 1.21$ (3H, d, J 6.8, C( $\left.\left.\alpha\right) M e\right), 1.24$ (9H, s, $\left.\mathrm{CMe}_{3}\right), 3.67\left(1 \mathrm{H}, \mathrm{d}, J\right.$ 15.3, $\left.\mathrm{NCH}_{\mathrm{A}} \mathrm{H}_{\mathrm{B}} \mathrm{Ar}\right), 3.86\left(1 \mathrm{H}, \mathrm{d}, J\right.$ 15.3, $\left.\mathrm{NCH}_{\mathrm{A}} H_{\mathrm{B}} \mathrm{Ar}\right), 4.18(1 \mathrm{H}, \mathrm{q}, J$ 6.8, $\mathrm{C}(\alpha) H)$, 4.21 (1H, d, J 3.2, C(3)H), 4.45 (1H, d, J 3.2, C(2)H), 6.21 (1H, d, J 0.9, C(4')H), 6.98-7.03 (2H, m, $\left.\mathrm{C}\left(3^{\prime \prime}\right) H, \mathrm{C}\left(5^{\prime \prime}\right) H\right), 7.24-7.48\left(9 \mathrm{H}, \mathrm{m}, \mathrm{C}\left(2^{\prime}\right) H, \mathrm{C}\left(5^{\prime}\right) H, \mathrm{C}\left(2^{\prime \prime}\right) H, \mathrm{C}\left(6^{\prime \prime}\right) H, P h\right) ; \delta_{\mathrm{C}}\left(125 \mathrm{MHz}, \mathrm{CDCl}_{3}\right) 13.7$ (C( $\alpha) M e), 27.7\left(\mathrm{CMe}_{3}\right), 42.5\left(\mathrm{NCH}_{2} \mathrm{Ar}\right), 56.7(C(\alpha)), 64.9(C(3))$, $73.1(C(2)), 82.4\left(C \mathrm{Me}_{3}\right), 110.6\left(C\left(4^{\prime}\right)\right)$, 114.9 (d, $J$ 21.0, $\left.C\left(3^{\prime \prime}\right), C\left(5^{\prime \prime}\right)\right), 125.3\left(C\left(3^{\prime}\right)\right), 126.9,127.8,128.2$ (o,m,p-Ph), 131.3 (d, $J$ 7.6, $C\left(2^{\prime \prime}\right)$, $\left.C\left(6^{\prime \prime}\right)\right), 133.9$ (d, J 2.9, $\left.C\left(1^{\prime \prime}\right)\right), 139.9\left(C\left(2^{\prime}\right)\right), 143.0$ (C(5')), 144.0 (i-Ph), 162.3 (d, $J$ 246.1, C(4")), 171.8 $(C(1)) ; \quad \delta_{\mathrm{F}}\left(377 \mathrm{MHz}, \mathrm{CDCl}_{3}\right)-114.8\left(\mathrm{C}\left(4^{\prime \prime}\right) \mathrm{F}\right) ; \mathrm{m} / \mathrm{z}\left(\mathrm{ESI}^{+}\right) 440\left([\mathrm{M}+\mathrm{H}]^{+}, 100 \%\right) ; \mathrm{HRMS}\left(\mathrm{ESI}^{+}\right)$ $\mathrm{C}_{26} \mathrm{H}_{31} \mathrm{FNO}_{4}{ }^{+}\left([\mathrm{M}+\mathrm{H}]^{+}\right)$requires 440.2232; found 440.2223 .

tert-Butyl (RS,RS,RS)-2-hydroxy-3-[N-(furan-3'-ylmethyl)- $N$-( $\alpha$-methylbenzyl)amino]-3- 
(4"-methoxyphenyl)propanoate 51: Following General procedure 3, s-BuLi (1.4 M in cyclohexane, $1.77 \mathrm{~mL}, 2.48 \mathrm{mmol}$ ) was reacted with 22 (515 mg, $2.56 \mathrm{mmol})$ and 40 (375 mg, $1.60 \mathrm{mmol},>95: 5 \mathrm{dr}$ [(E):(Z)]) in THF (6.41 mL) before the addition of 14 (624 mg, $2.72 \mathrm{mmol})$. Purification via flash column chromatography (eluent $30-40{ }^{\circ} \mathrm{C}$ petrol/ $/ \mathrm{Et}_{2} \mathrm{O} / 35 \%$ aqNH$\left.{ }_{4} \mathrm{OH}, 90: 9: 1\right)$ gave $\mathbf{5 1}$ as a pale yellow oil (277 mg, 38\%, 88:12 dr); $v_{\max }(\mathrm{ATR}) 3635(\mathrm{O}-\mathrm{H}), 1725(\mathrm{C}=\mathrm{O}) ; \delta_{\mathrm{H}}\left(400 \mathrm{MHz}, \mathrm{CDCl}_{3}\right)$ [selected peaks for the major diastereoisomer] 1.20 (3H, d, $J$ 6.8, C( $\alpha) M e$ ), 1.24 (9H, s, CMe3), 3.63 (1H, d, $J 15.2, \mathrm{NCH}_{\mathrm{A}} \mathrm{H}_{\mathrm{B}} \mathrm{Ar}$ ), $3.80(3 \mathrm{H}, \mathrm{s}, \mathrm{OMe}), 3.85\left(1 \mathrm{H}, \mathrm{d}, J\right.$ 15.2, $\left.\mathrm{NCH}_{\mathrm{A}} H_{\mathrm{B}} \mathrm{Ar}\right), 4.17(1 \mathrm{H}, \mathrm{d}, J 3.2, \mathrm{C}(3) H), 4.19(1 \mathrm{H}, \mathrm{q}, J$ 6.8, $\mathrm{C}(\alpha) H)$, $4.43(1 \mathrm{H}, \mathrm{d}, J 3.2, \mathrm{C}(2) H), 6.20(1 \mathrm{H}, \mathrm{dd}, J$ 1.7, 0.7, C(4')H), 6.83-6.87 (2H, m, C(3")H, C(5")H); $\delta_{\mathrm{H}}\left(400 \mathrm{MHz}, \mathrm{CDCl}_{3}\right)$ [selected peaks for the minor diasteroisomer] 1.27 (9H, s, CMe $), 1.44$ (3H, d, $J$ 6.8, $\mathrm{C}(\alpha) M e$ ), 3.78 (3H, s, OMe), 4.00 (1H, dd, J 15.9, $\left.\mathrm{NCH}_{\mathrm{A}} H_{\mathrm{B}} \mathrm{Ar}\right), 4.62$ (1H, d, $J$ 3.2, C(2)H); $\delta_{\mathrm{C}}(100 \mathrm{MHz}$, $\left.\mathrm{CDCl}_{3}\right)$ [major diastereoisomer] $13.4(\mathrm{C}(\alpha) M e), 27.8\left(\mathrm{CMe}_{3}\right), 42.5\left(\mathrm{NCH}_{2} \mathrm{Ar}\right), 55.2(\mathrm{OMe}), 56.6(C(\alpha))$, 65.3 (C(3)), $73.3(C(2)), 82.1\left(C \mathrm{Me}_{3}\right), 110.6\left(C\left(4^{\prime}\right)\right), 113.4\left(C\left(3^{\prime \prime}\right), C\left(5^{\prime \prime}\right)\right), 125.4\left(C\left(3^{\prime}\right)\right), 126.7,127.9$,

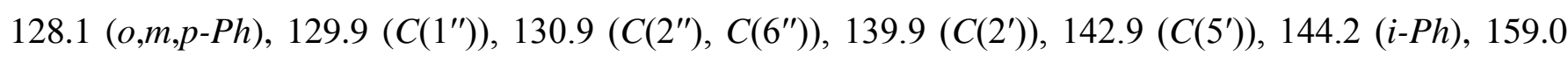
$\left(C\left(4^{\prime \prime}\right)\right), 171.9(C(1)) ; \mathrm{m} / \mathrm{z}\left(\mathrm{ESI}^{+}\right) 452\left([\mathrm{M}+\mathrm{H}]^{+}, 100 \%\right) ; \mathrm{HRMS}\left(\mathrm{ESI}^{+}\right) \mathrm{C}_{27} \mathrm{H}_{34} \mathrm{NO}_{5}{ }^{+}\left([\mathrm{M}+\mathrm{H}]^{+}\right)$requires 452.2431; found 452.2426.

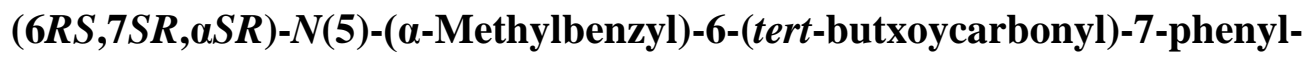

4,5,6,7-tetrahydrothieno[3,2-c]pyridine 55: Following General procedure 4, $\mathrm{Tf}_{2} \mathrm{O}$ (194 mg, 0.686 mmol) was reacted with 45 (200 mg, $0.46 \mathrm{mmol}$, >95:5 dr) and 2,6-di-tert-butyl-4-methylpyridine (281 mg, $1.37 \mathrm{mmol}$ ) in $\mathrm{CH}_{2} \mathrm{Cl}_{2}(3.81 \mathrm{~mL})$. Purification via flash column chromatography (eluent $30-40{ }^{\circ} \mathrm{C}$ petrol/Et ${ }_{2} \mathrm{O} / 35 \%$ aqNH$\left.{ }_{4} \mathrm{OH}, 90: 9: 1\right)$ gave 55 as a pale yellow oil (80 mg, 42\%, >95:5 dr); $v_{\max }$ (ATR) $1722(\mathrm{C}=\mathrm{O}) ; \delta_{\mathrm{H}}\left(400 \mathrm{MHz}, \mathrm{CDCl}_{3}\right) 1.13(3 \mathrm{H}, \mathrm{d}, J$ 6.6, C( $\left.\alpha) M e\right), 1.41$ (9H, s, CMe 3 ), 3.67 (1H, d, J 15.2,

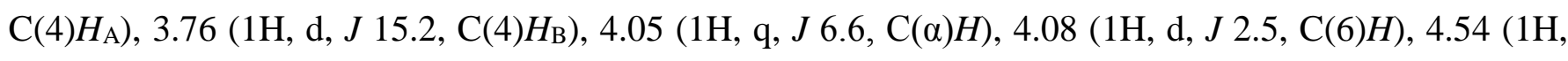
app s, C(7)H), 6.62 (1H, d, J 5.1, C(3)H), 7.07 (1H, d, J 5.1, C(2)H), 7.15-7.35 (10H, m, Ph); $\delta_{\mathrm{C}}(100$ MHz, $\left.\mathrm{CDCl}_{3}\right) 21.3(\mathrm{C}(\alpha) M e), 28.2\left(\mathrm{CMe}_{3}\right), 44.6$ (C(7)), $47.6(C(4)), 61.8(C(\alpha)), 63.5(C(6)), 81.4$ (CMe $), 123.8$ (C(2)), 124.6 (C(3)), 126.7, 126.8, 127.1, 127.9, 128.3, 128.4 (o,m,p-Ph), 133.9 (C(7a)), 135.1 (C(3a)), 144.0, $146.2(\mathrm{i}-\mathrm{Ph}), 171.4\left(\mathrm{CO}_{2}{ }^{\mathrm{B}} \mathrm{Bu}\right) ; \mathrm{m} / \mathrm{z}\left(\mathrm{ESI}^{+}\right) 420\left([\mathrm{M}+\mathrm{H}]^{+}, 100 \%\right) ; \mathrm{HRMS}\left(\mathrm{ESI}^{+}\right)$ $\mathrm{C}_{26} \mathrm{H}_{30} \mathrm{NO}_{2} \mathrm{~S}^{+}\left([\mathrm{M}+\mathrm{H}]^{+}\right)$requires 420.1992; found 420.1988 .

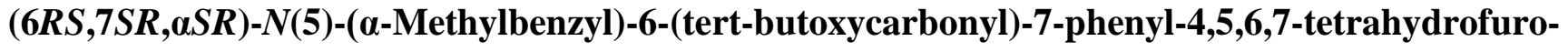

[3,2-c]pyridine 56: Following General procedure 4, Tf $2 \mathrm{O}$ (302 mg, $1.07 \mathrm{mmol}$ ) was reacted with 47 (300 mg, $0.71 \mathrm{mmol},>95: 5 \mathrm{dr}$ ) and 2,6-di-tert-butyl-4-methylpyridine (438 mg, $2.14 \mathrm{mmol})$ in $\mathrm{CH}_{2} \mathrm{Cl}_{2}(8.91$ $\mathrm{mL}$ ). Purification via flash column chromatography (eluent $30-40{ }^{\circ} \mathrm{C}$ petrol/ $\mathrm{Et}_{2} \mathrm{O} / 35 \%$ aqNH $\mathrm{OH}_{4}$, 90:9:1) gave 56 as a pale yellow oil (172 mg, 60\%, >95:5 dr); $v_{\max }(\mathrm{ATR}) 1726$ (C=O); $\delta_{\mathrm{H}}(500 \mathrm{MHz}$, $\left.\mathrm{CDCl}_{3}\right) 1.16(3 \mathrm{H}, \mathrm{d}, J$ 6.6, C( $\left.\alpha) M e\right), 1.47$ (9H, s, CMe $), 3.52\left(1 \mathrm{H}, \mathrm{d}, J\right.$ 14.4, C(4)H $\left.\mathrm{A}_{\mathrm{A}}\right), 3.64(1 \mathrm{H}, \mathrm{d}, J$ 14.4, C(4)HB $), 4.07(1 \mathrm{H}, \mathrm{d}, J$ 2.0, C(6)H), $4.08(1 \mathrm{H}, \mathrm{q}, J$ 6.6, C( $(\alpha) H), 4.54(1 \mathrm{H}, \mathrm{app} \mathrm{s}, \mathrm{C}(7) H), 6.11(1 \mathrm{H}, \mathrm{d}, J$ 
1.7, C(3)H), 7.19-7.38 (11H, m, C(2)H, Ph); $\delta_{\mathrm{C}}\left(125 \mathrm{MHz}, \mathrm{CDCl}_{3}\right) 21.7(\mathrm{C}(\alpha) M e), 28.2\left(\mathrm{CMe}_{3}\right), 43.4$ (C(7)), 44.6 (C(4)), $61.9(C(\alpha)), 63.7$ (C(6)), $81.4\left(C \mathrm{Me}_{3}\right), 108.1$ (C(3)), 117.1 (C(3a)), 126.8, 127.0, 128.1, 128.3 (o,m,p-Ph), $141.7(C(2)), 141.8$ (i-Ph), $146.4(i-P h), 148.3(C(7 \mathrm{a})), 171.4\left(C \mathrm{O}_{2}{ }^{\mathrm{t}} \mathrm{Bu}\right) ; \mathrm{m} / \mathrm{z}$ $\left(\mathrm{ESI}^{+}\right) 404\left([\mathrm{M}+\mathrm{H}]^{+}, 100 \%\right)$; HRMS $\left(\mathrm{ESI}^{+}\right) \mathrm{C}_{26} \mathrm{H}_{30} \mathrm{NO}_{3}{ }^{+}\left([\mathrm{M}+\mathrm{H}]^{+}\right)$requires 404.2220; found 404.2217.

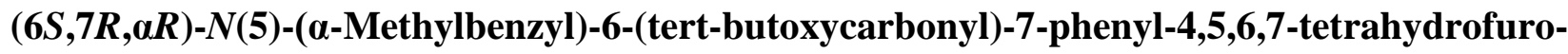

[3,2-c]pyridine 56: Following General procedure 4, $\mathrm{Tf}_{2} \mathrm{O}(0.12 \mathrm{~mL}, 0.71 \mathrm{mmol})$ was reacted with (R,R,R)-47 (200 mg, $0.48 \mathrm{mmol}$, >95:5 dr) and 2,6-di-tert-butyl-4-methylpyridine (292 mg, $1.43 \mathrm{mmol}$ ) in $\mathrm{CH}_{2} \mathrm{Cl}_{2}(5.94 \mathrm{~mL})$. Purification via flash column chromatography (eluent $30-40{ }^{\circ} \mathrm{C}$ petrol/Et $2 \mathrm{O} / 35 \%$ aqNH$\left.{ }_{4} \mathrm{OH}, 90: 9: 1\right)$ gave $(6 S, 7 R, \alpha R)-56$ as a pale yellow oil (123 mg, 64\%, >95:5 dr); $[\alpha]_{\mathrm{D}}^{22}-12.8(c 1.0$ in $\left.\mathrm{CHCl}_{3}\right)$.

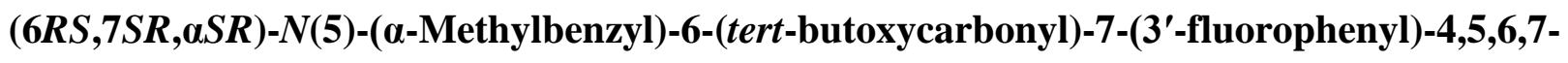

tetrahydrofuro[3,2-c]pyridine 57: Following General procedure 4, $\mathrm{Tf}_{2} \mathrm{O}$ (260 mg, $0.92 \mathrm{mmol}$ ) was reacted with 48 (270 mg, $0.62 \mathrm{mmol}$, >95:5 dr) and 2,6-di-tert-butyl-4-methylpyridine (378 mg, 1.85 mmol) in $\mathrm{CH}_{2} \mathrm{Cl}_{2}(7.69 \mathrm{~mL})$. Purification via flash column chromatography (eluent $30-40{ }^{\circ} \mathrm{C}$ petrol/Et $2 \mathrm{O} / 35 \%$ aqNH $\left.4{ }_{4} \mathrm{OH}, 90: 9: 1\right)$ gave 57 as a pale yellow oil (199 mg, 77\%, >95:5 dr); $v_{\max }$ (ATR) $1725(\mathrm{C}=\mathrm{O}) ; \delta_{\mathrm{H}}\left(500 \mathrm{MHz}, \mathrm{CDCl}_{3}\right) 1.17$ (3H, d, J 6.7, C( $\left.\left.\alpha\right) M e\right), 1.47$ (9H, s, CMe $)$ ), 3.52 (1H, d, J 14.4,

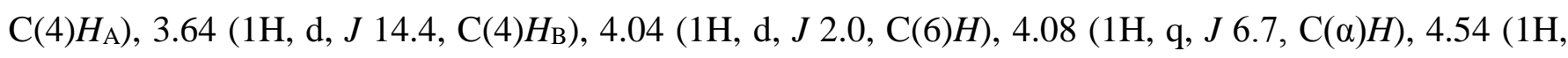
app s, C(7)H), 6.12 (1H, d, J 2.0, C(3)H), 6.96-7.38 (10H, m, Ph, C(2)H, C(2')H, C(4')H, C(5')H, $\left.\mathrm{C}\left(6^{\prime}\right) H\right)$; $\delta_{\mathrm{C}}\left(125 \mathrm{MHz}, \mathrm{CDCl}_{3}\right) 21.6(\mathrm{C}(\alpha) M e), 28.2$ (CMes), $43.1(C(7)), 44.6(C(4)), 61.9(C(\alpha)), 63.4$ (C(6)), $81.7\left(C \mathrm{Me}_{3}\right), 108.2$ (C(3)), 113.8 (d, J 21.0, C(4')), 115.4 (d, J 21.9, C(2')), 117.4 (C(3a)), 123.9 (d, J 2.9, $C\left(6^{\prime}\right)$ ), 126.9, 127.1, 128.4 (o,m,p-Ph), 129.4 (d, J 7.6, C(5')), $141.9(C(2)), 144.5$ (d, J 7.6, C(1')), 146.1 (i-Ph), 147.8 (C(7a)), 162.8 (d, $J$ 245.1, $\left.C\left(3^{\prime}\right)\right), 171.2\left(\mathrm{CO}_{2}{ }^{\mathrm{t}} \mathrm{Bu}\right) ; \delta_{\mathrm{F}}\left(377 \mathrm{MHz}, \mathrm{CDCl}_{3}\right)-113.9$ $\left(\mathrm{C}\left(3^{\prime}\right) \mathrm{F}\right) ; \mathrm{m} / \mathrm{z}\left(\mathrm{ESI}^{+}\right) 422\left([\mathrm{M}+\mathrm{H}]^{+}, 100 \%\right) ; \mathrm{HRMS}\left(\mathrm{ESI}^{+}\right) \mathrm{C}_{26} \mathrm{H}_{29} \mathrm{FNO}_{3}{ }^{+}\left([\mathrm{M}+\mathrm{H}]^{+}\right)$requires 422.2126; found 422.2124 .

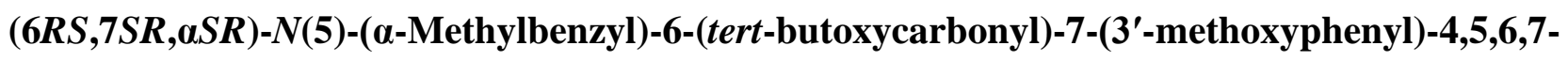

tetrahydrofuro[3,2-c]pyridine 58: Following General procedure 4, $\mathrm{Tf}_{2} \mathrm{O}$ (216 $\mathrm{mg}, 0.77 \mathrm{mmol}$ ) was reacted with 49 (230 mg, $0.51 \mathrm{mmol}$, >95:5 dr) and 2,6-di-tert-butyl-4-methylpyridine (314 mg, 1.53 mmol) in $\mathrm{CH}_{2} \mathrm{Cl}_{2}(6.37 \mathrm{~mL})$. Purification via flash column chromatography (eluent $30-40{ }^{\circ} \mathrm{C}$ petrol/Et ${ }_{2} \mathrm{O} / 35 \%$ aqNH$\left.{ }_{4} \mathrm{OH}, 90: 9: 1\right)$ gave 58 as a pale yellow oil (179 mg, 81\%, >95:5 dr); $v_{\max }$ (ATR) $1725(\mathrm{C}=\mathrm{O}) ; \delta_{\mathrm{H}}\left(500 \mathrm{MHz}, \mathrm{CDCl}_{3}\right) 1.19$ (3H, d, J 6.6, C( $\left.\left.\alpha\right) M e\right), 1.47$ (9H, s, CMe 3 ), 3.52 (1H, d, J 14.5, $\left.\mathrm{C}(4) H_{\mathrm{A}}\right), 3.64(1 \mathrm{H}$, app d, $J$ 14.5, C(4)HB $), 3.82$ (3H, s, OMe), 4.08 (1H, d, $J$ 1.9, C(6)H), $4.08(1 \mathrm{H}, \mathrm{q}, J$ 6.6, C $(\alpha) H), 4.51(1 \mathrm{H}$, app s, C(7)H), $6.10(1 \mathrm{H}, \mathrm{d}, J$ 1.9, C(3)H), 6.82-7.29 (10H, m, C(2)H, C(2')H, $\left.\mathrm{C}\left(4^{\prime}\right) H, \mathrm{C}\left(5^{\prime}\right) H, \mathrm{C}\left(6^{\prime}\right) H, P h\right) ; \delta_{\mathrm{C}}\left(125 \mathrm{MHz}, \mathrm{CDCl}_{3}\right) 21.8(\mathrm{C}(\alpha) M e), 28.2\left(\mathrm{CMe}_{3}\right), 43.4(C(7))$, $44.6(C(4))$, 
55.2 (OMe), $61.9(C(\alpha)), 63.6$ (C(6)), 81.5 (CMe3), $108.1(C(3)), 112.3\left(C\left(4^{\prime}\right)\right), 114.0\left(C\left(2^{\prime}\right)\right), 117.1$ (C(3a)), $120.8\left(C\left(6^{\prime}\right)\right), 126.8,127.1,128.3$ (o,m,p-Ph), $129.0\left(C\left(5^{\prime}\right)\right), 141.7(C(2)), 143.5\left(C\left(1^{\prime}\right)\right), 143.5$ (i-Ph), 148.2 (C(7a)), $159.5\left(C\left(3^{\prime}\right)\right), 171.4\left(\mathrm{CO}_{2}{ }^{\mathrm{t}} \mathrm{Bu}\right) ; \mathrm{m} / \mathrm{z}\left(\mathrm{ESI}^{+}\right) 434\left([\mathrm{M}+\mathrm{H}]^{+}, 100 \%\right) ; \mathrm{HRMS}\left(\mathrm{ESI}^{+}\right)$ $\mathrm{C}_{27} \mathrm{H}_{32} \mathrm{NO}_{4}{ }^{+}\left([\mathrm{M}+\mathrm{H}]^{+}\right)$requires 434.2326; found 434.2321.

(6RS,7SR, $\alpha S R)-N(5)-(\alpha-M e t h y l b e n z y l)-6-(t e r t-b u t o x y c a r b o n y l)-7-\left(4^{\prime}\right.$-fluorophenyl)-4,5,6,7-

tetrahydrofuro[3,2-c]pyridine 59: Following General procedure 4, $\mathrm{Tf}_{2} \mathrm{O}$ (183 mg, $0.65 \mathrm{mmol}$ ) was reacted with 50 (190mg, 0.43 mmol, 94:6 dr) and 2,6-di-tert-butyl-4-methylpyridine (266 mg, 1.30 $\mathrm{mmol}$ ) in $\mathrm{CH}_{2} \mathrm{Cl}_{2}(5.41 \mathrm{~mL})$. Purification via flash column chromatography (eluent $30-40{ }^{\circ} \mathrm{C}$ petrol/Et $2 \mathrm{O} / 35 \%$ aqNH$\left.{ }_{4} \mathrm{OH}, 90: 9: 1\right)$ gave 59 as a pale yellow oil (140 mg, 77\%, >95:5 dr); $v_{\max }$ (ATR) $1725(\mathrm{C}=\mathrm{O}) ; \delta_{\mathrm{H}}\left(500 \mathrm{MHz}, \mathrm{CDCl}_{3}\right) 1.16(3 \mathrm{H}, \mathrm{d}, J$ 6.6, C( $\left.\alpha) M e\right), 1.47$ (9H, s, CMe $)$ ), 3.51 (1H, d, J 14.5, C(4) $\left.H_{\mathrm{A}}\right), 3.64(1 \mathrm{H}$, app d, $J$ 14.5, C(4)HB $), 3.99(1 \mathrm{H}, \mathrm{d}, J 1.9, \mathrm{C}(6) H), 4.08(1 \mathrm{H}, \mathrm{q}, J 6.6, \mathrm{C}(\alpha) H), 4.52$ (1H, app s, C(7)H), 6.11 (1H, d, $J$ 1.9, C(3)H), 7.01-7.29 (10H, m, C(2)H, C(2')H, C(3')H, C(5')H, C(6')H, $P h) ; \delta_{\mathrm{C}}\left(125 \mathrm{MHz}, \mathrm{CDCl}_{3}\right) 21.6(\mathrm{C}(\alpha) M e), 28.2\left(\mathrm{CMe}_{3}\right), 42.6(C(7)), 44.6(C(4)), 61.9(C(\alpha)), 63.7(C(6))$, $81.6\left(C \mathrm{Me}_{3}\right), 108.2$ (C(3)), 114.8 (d, $J$ 21.0, $\left.C\left(3^{\prime}\right), C\left(5^{\prime}\right)\right), 117.2$ (C(3a)), 126.9, 127.1, 128.4 (o,m,p-Ph), 129.8 (d, $J$ 8.6, $\left.C\left(2^{\prime}\right), C\left(6^{\prime}\right)\right), 137.5$ (d, $J$ 2.9, $\left.C\left(1^{\prime}\right)\right), 141.8$ (C(2)), 146.2 (i-Ph), 148.2 (C(7a)), 161.9 (d, $J$ 245.1, $\left.C\left(4^{\prime}\right)\right), 171.2\left(\mathrm{CO}_{2}{ }^{\mathrm{t}} \mathrm{Bu}\right) ; \delta_{\mathrm{F}}\left(377 \mathrm{MHz}, \mathrm{CDCl}_{3}\right)-116.4\left(\mathrm{C}\left(4^{\prime}\right) F\right) ; \mathrm{m} / \mathrm{z}\left(\mathrm{ESI}^{+}\right) 422\left([\mathrm{M}+\mathrm{H}]^{+}, 100 \%\right)$; HRMS $\left(\mathrm{ESI}^{+}\right) \mathrm{C}_{26} \mathrm{H}_{29} \mathrm{FNO}_{3}{ }^{+}\left([\mathrm{M}+\mathrm{H}]^{+}\right)$requires 422.2126; found 422.2122.

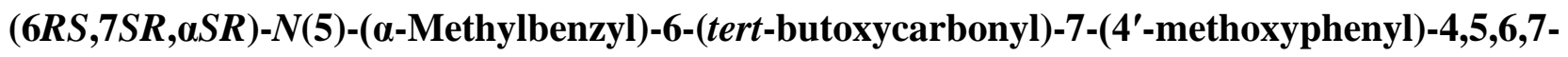

tetrahydrofuro[3,2-c]pyridine-6-carboxylate 60: Following General procedure 4, $\mathrm{Tf}_{2} \mathrm{O}$ (234 mg, 0.83 mmol) was reacted with 51 (250mg, 0.55 mmol, 88:12 dr) and 2,6-di-tert-butyl-4-methylpyridine (341 mg, $1.66 \mathrm{mmol}$ ) in $\mathrm{CH}_{2} \mathrm{Cl}_{2}(6.93 \mathrm{~mL})$. Purification via flash column chromatography (eluent $30-40{ }^{\circ} \mathrm{C}$ petrol/Et ${ }_{2} \mathrm{O} / 35 \%$ aqNH$\left.{ }_{4} \mathrm{OH}, 90: 9: 1\right)$ gave $\mathbf{6 0}$ as a pale yellow oil (150 mg, 63\%, 88:12 dr); $v_{\max }$ (ATR) $1724(\mathrm{C}=\mathrm{O}) ; \delta_{\mathrm{H}}\left(500 \mathrm{MHz}, \mathrm{CDCl}_{3}\right)$ [selected peaks for the major diastereoisomer] $1.18(3 \mathrm{H}, \mathrm{d}, J$ 6.8, $\mathrm{C}(\alpha) M e), 1.47$ (9H, s, CMe 3 ), 3.51 (1H, d, $J$ 14.5, C(4)H $\left.H_{\mathrm{A}}\right), 3.63(1 \mathrm{H}$, app dd, $J$ 14.5, C(4)HB), $3.83(3 \mathrm{H}, \mathrm{s}$, OMe), 4.02 (1H, d, $J$ 1.9, C(6)H), $4.08(1 \mathrm{H}, \mathrm{q}, J$ 6.8, C( $\alpha) H), 4.48(1 \mathrm{H}$, app s, C(7)H), $6.10(1 \mathrm{H}, \mathrm{d}, J 1.9$, $\mathrm{C}(3) H) ; \delta_{\mathrm{H}}\left(500 \mathrm{MHz}, \mathrm{CDCl}_{3}\right.$ ) [selected peaks for the minor diastereoisomer] $1.36(3 \mathrm{H}, \mathrm{d}, J$ 6.6, $\mathrm{C}(\alpha) \mathrm{Me})$, 1.41 (9H, s, CMe 3 ), 3.41 (1H, d, J 1.9, C(6)H), 4.17 (1H, q, J 6.6, C $(\alpha) H), 4.31$ (1H, app s, C(7)H), 6.30 $(1 \mathrm{H}, \mathrm{d}, J 1.9, \mathrm{C}(3) H)$; $\delta_{\mathrm{C}}\left(125 \mathrm{MHz}, \mathrm{CDCl}_{3}\right)$ [selected peaks for the major diastereoisomer] $21.6(\mathrm{C}(\alpha) M e)$, 28.2 (CMes), $42.6(C(7))$, $44.6(C(4)), 55.2$ (OMe), $61.9(C(\alpha)), 63.9(C(6)), 81.4\left(C \mathrm{Me}_{3}\right), 108.1(C(3))$, $113.5\left(C\left(3^{\prime}\right), C\left(5^{\prime}\right)\right), 116.8$ (C(3a)), 126.8, 127.1, 128.3 (o,m,p-Ph), $129.3\left(C\left(2^{\prime}\right), C\left(6^{\prime}\right)\right), 134.0\left(C\left(1^{\prime}\right)\right)$, $141.6(C(2)), 146.4(\mathrm{i}-\mathrm{Ph}), 148.7(C(7 \mathrm{a})), 154.5\left(C\left(4^{\prime}\right)\right), 171.5\left(\mathrm{CO}_{2}{ }^{\mathrm{t}} \mathrm{Bu}\right) ; \delta_{\mathrm{C}}\left(125 \mathrm{MHz}, \mathrm{CDCl}_{3}\right)$ [selected peaks for the minor diastereoisomer] $22.4(\mathrm{C}(\alpha) M e), 28.2$ (CMes), $42.6(C(4)), 42.7(C(7)), 55.4(\mathrm{OMe})$, $61.4(C(\alpha)), 65.2(C(6)), 81.1\left(C \mathrm{Me}_{3}\right), 108.3(C(3)), 113.3\left(C\left(3^{\prime}\right), C\left(5^{\prime}\right)\right), 117.3(C(3 a)), 126.6,127.2$, 128.0 (o,m,p-Ph), $128.6\left(C\left(2^{\prime}\right), C\left(6^{\prime}\right)\right), 133.6\left(C\left(1^{\prime}\right)\right), 141.8(C(2)), 145.1(i-P h), 148.6(C(7 a)), 158.4$ 
$\left(C\left(4^{\prime}\right)\right), 171.3\left(\mathrm{CO}_{2}{ }^{\mathrm{t}} \mathrm{Bu}\right) ; \mathrm{m} / \mathrm{z}\left(\mathrm{ESI}^{+}\right) 434\left([\mathrm{M}+\mathrm{H}]^{+}, 100 \%\right) ; \mathrm{HRMS}\left(\mathrm{ESI}^{+}\right) \mathrm{C}_{27} \mathrm{H}_{32} \mathrm{NO}_{4}{ }^{+}\left([\mathrm{M}+\mathrm{H}]^{+}\right)$requires 434.2326; found 434.2320.

\section{REFERENCES AND NOTES}

1. S. Tsukamoto, Y. Nagasawa, R. Ueoka, R. Yamanokuchi, N. Horiuchi and T. Ikeda, Chem. Pham. Bull., 2011, 59, 287.

2. (a) W. J. Giardina and M. Williams, CNS Drug Rev., 2001, 7, 305; (b) Y. G. Gu, E. K. Bayburt, M. R. Michaelides, C. W. Lin and K. Shiosaki, Bioorg. Med. Chem. Lett., 1999, 9, 1341; (c) S. Hajra and S. Bar, Chem. Commun., 2011, 47, 3981.

3. S. Chowdhury, K. N. Owens, R. J. Herr, Q. Jiang, X. Chen, G. Johnson, V. E. Groppi, D. W. Raible, E. W. Rubel and J. A. Simon, J. Med. Chem., 2018, 61, 84.

4. S. G. Davies, A. M. Fletcher, A. B. Frost, M. S. Kennedy, P. M. Roberts and J. E. Thomson, Tetrahedron, 2016, 72, 2139.

5. For examples of diastereoselective aminohydroxylation methodology, see: M. E. Bunnage, S. G. Davies, C. J. Goodwin and O. Ichihara, Tetrahedron, 1994, 50, 3975 and ref 10.

6. $\quad$ S. G. Davies, A. M. Fletcher and P. M. Roberts, Org. Synth., 2010, 87, 143.

7. (a) J. F. Costello, S. G. Davies and O. Ichihara, Tetrahedron: Asymmetry, 1994, 5, 1999; (b) T. D. W. Claridge, S. G. Davies, D. Kruchinin, B. Odell, P. M. Roberts, A. J. Russell, J. E. Thomson and S. M. Toms, Tetrahedron: Asymmetry, 2013, 24, 947.

8. S. D. Bull, S. G. Davies, D. J. Fox, M. Gianotti, P. M. Kelly, C. Pierres, E. D. Savory, and A. D. Smith, J. Chem. Soc., Perkin Trans. 1, 2002, 1858.

9. (a) S. G. Davies, A. D. Smith and P. D. Price, Tetrahedron: Asymmetry, 2005, 16, 2833; (b) S. G. Davies, A. M. Fletcher, P. M. Roberts and J. E. Thomson, Tetrahedron: Asymmetry, 2012, 23, 1111; (c) S. G. Davies, A. M. Fletcher, P. M. Roberts and J. E. Thomson, Tetrahedron: Asymmetry, 2017, 28, 1842.

10. M. E. Bunnage, S. G. Davies, C. J. Goodwin and O. Ichihara, Tetrahedron, 1994, 50, 3975.

11. (a) S. G. Davies, A. M. Fletcher, J. A. Lee, P. M. Roberts, M. Y. Souleymanou, J. E. Thomson and C. M. Zammit, Org. Biomol. Chem., 2014, 12, 2702; (b) S. G. Davies, E. C. Goddard, P. M. Roberts, A. J. Russell, and J. E. Thomson, Synlett, 2015, 1541; (c) S. G. Davies, N. M. Garrido, D. Kruchinin, O. Ichihara, L. J. Kotchie, P. D. Price, A. J. Price Motrimer, A. J. Russell and A. D. Smith, Tetrahedron: Asymmetry, 2006, 17, 1793; (d) S. G. Davies, A. W. Mulvaney, A. J. Russell and A. D. Smith, Tetrahedron: Asymmetry, 2007, 18, 1554; (e) S. G. Davies, J. A. Lee, P. M. Roberts, J. P. Stonehouse and J. E. Thomson, Tetrahedron Lett., 2012, 53, 1119.

12. S. G. Davies, E. M. Foster, C. R. McIntosh, P. M. Roberts, T. E. Rosser, A. D. Smith and J. E. 
Thomson, Tetrahedron: Asymmetry, 2011, 22, 1035.

13. (a) S. D. Bull, S. G. Davies, G. Fenton, A. W. Mulvany, R. S. Prasad and A. D. Smith, J. Chem. Soc., Perkin Trans. 1, 2000, 3765; (b) S. D. Bull, S. G. Davies, G. Fenton, A. W. Mulvany, R. S. Prasad and A. D. Smith, Chem. Commun., 2000, 337.

14. Typically ${ }^{1} \mathrm{H}$ NMR ${ }^{3} J$ coupling constants of $\sim 4 \mathrm{~Hz}$ were observed between the $\mathrm{C}(2) H$ and $\mathrm{C}(3) H$ protons of 2,3-anti- $\alpha$-hydroxy- $\beta$-amino esters, whereas ${ }^{1} \mathrm{H}$ NMR ${ }^{3} J$ coupling constants for the corresponding 2,3-syn- $\alpha$-hydroxy- $\beta$-amino esters are known to be typically $\sim 10 \mathrm{~Hz}$.

15. S. G. Davies, A. M. Fletcher, A. B. Frost, P. M. Roberts and J. E. Thomson, Tetrahedron, 2014, 72, 2139.

16. The ${ }^{1} \mathrm{H}$ NMR ${ }^{3} J$ coupling constants between the $\mathrm{C}(3) H$ and $\mathrm{C}(4) H$ protons of 1,2,3,4-tetrahedroisoquinolines are known to be diagnostic, with ${ }^{3} J_{3,4}=\sim 3 \mathrm{~Hz}$ for 3,4-anti-tetrahydroisoquinolines and ${ }^{3} J_{3,4}=\sim 6 \mathrm{~Hz}$ for 3,4-syn-tetrahydroisoquinolines being observed; see: ref 15.

17. J. A. Joule and K. Mills 'Heterocyclic Chemistry', Blackwell Science Ltd., Oxford, 2000, pp 233236.

18. A. B. Pangborn, M. A. Giardello, R. H. Grubbs, P. K. Rosen and F. J. Timmers, Organometallics, 1996, 15, 1518.

19. F. Gautier, S. Jones, X. Lia and S. J. Martin, Org. Biomol. Chem., 2011, 9, 7860.

20. P. Kang, K. M. Lee, W. K. Lee, K. H. Lee, B. Lee, J. Choc and N. H. Hur, RSC Adv., 2014, 4, 46203.

21. S. A. Sergeyev and M. Hesse, Helv. Chim. Acta, 2002, 85, 161. 